\title{
IRAS 18511+0146: a proto Herbig Ae/Be cluster?`
}

\author{
S. Vig ${ }^{1}$, L. Testi ${ }^{1,2}$, M. Walmsley ${ }^{1}$, S. Molinari ${ }^{3}$, S. Carey ${ }^{4}$, and A. Noriega-Crespo ${ }^{4}$ \\ 1 INAF-Osservatorio Astrofisico di Arcetri, Largo E. Fermi 5, 50125 Firenze, Italy \\ e-mail: sarita@arcetri.astro.it \\ 2 ESO, Karl Schwarzschild str. 2, 85748 Garching, Germany \\ 3 INAF - Istituto di Fisica dello Spazio Interplanetario, Via Fosso del Cavaliere, 00133 Roma, Italy \\ ${ }^{4}$ Spitzer Science Center, California Institute of Technology, Pasadena, CA 91125, USA
}

Received 2 April 2007 / Accepted 18 May 2007

\section{ABSTRACT}

\begin{abstract}
Context. The evolution of a young protocluster depends on the relative spatial distribution and dynamics of both stars and gas. Aims. We study the distribution and properties of the gas and stars surrounding the luminous $\left(10^{4} L_{\odot}\right)$ protocluster IRAS $18511+0146$. Methods. IRAS 18511+0146 and the cluster associated with it has been investigated using the sub-millimetre (JCMT-SCUBA), infrared (Spitzer-MIPSGAL, Spitzer-GLIMPSE, Palomar) and radio (VLA) continuum data. Cluster simulations have been carried out in order to understand the properties of clusters as well as to compare with the observations.

Results. The central most obscured part of the protocluster coincident with the compact sub-millimetre source found with SCUBA is responsible for at least $2 / 3$ of the total luminosity. A number of cluster members have been identified which are bright in mid infrared and show rising (near to mid infrared) spectral energy distributions suggesting that these are very young stellar sources. In the mid infrared $8.0 \mu \mathrm{m}$ image, a number of filamentary structures and clumps are detected in the vicinity of IRAS $18511+0146$.

Conclusions. Based on the luminosity and cluster size as well as on the evolutionary stages of the cluster members, IRAS $18511+0146$ is likely to be protocluster with the most massive object being a precursor to a Herbig type star.
\end{abstract}

Key words. stars: formation - stars: pre-main sequence - infrared: ISM - submillimeter - stars: individual: IRAS 18511+0146

\section{Introduction}

Massive stars form accompanied by swarms of lower mass objects and the relationship between the two is by no means clear. It is however known that the development of a young cluster depends sensitively on events which occur in the phase when it is still surrounded by the remnants of the "core" from which it formed (Lada \& Lada 2003). It is therefore of interest to obtain sensitive observations of the young protostars and the surrounding gas in the phase when dust obscuration causes the cluster to be invisible at optical wavelengths. The objects of interest are thus best observed at infrared and radio wavelengths.

This article reports such a study of the cluster surrounding what appears to be a young intermediate $\left(2-8 M_{\odot}\right)$ protostar (IRAS 18511+0146, also known as Mol 75 and RAFGL 5542) from the survey of Molinari et al. $(1996,1998)$ using both data from the Spitzer satellite and ground based observations. We proceed on the hypothesis that the cluster surrounding IRAS $18511+0146$ is essentially a forerunner of the small clusters examined by Testi et al. (1998). Thus, the bolometric luminosity (roughly $\left.10^{4} L_{\odot}\right)$ and size $(0.5$ parsec) are similar but, as discussed later, the visual extinction is large (of order 50 visual magnitudes) and variable.

The gas distribution is of great importance both because of its effect upon the extinction to individual protostars and because the gas plays an important role dynamically. The outflows and ionization caused by the young protostars are thought to be the primary agent causing the cluster to disperse. We therefore use

$\star$ Appendices A and B are only available in electronic form at http://www . aanda. org
JCMT-SCUBA observations to assess the mass and distribution of the gas associated with IRAS $18511+0146$. We then use this information in simulations which we have carried out with the aim of testing our hypothesis that the young protocluster associated with IRAS $18511+0146$ is an obscured version of the intermediate mass clusters studied by Testi et al. (1998).

Previous studies of IRAS 18511 were carried out by Watt \& Mundy (1999) using the OVRO interferometer and the VLA to map both molecular line and continuum emission. They detected what appeared to be a small ionized region ionized by a B1 star which coincided with a compact clump seen in $\mathrm{C}^{18} \mathrm{O}$. Kurtz et al. (2004) detected a methanol maser which was however offset by roughly $0.4 \mathrm{pc}\left(\sim 19^{\prime \prime}\right)$ relative to the ionized gas for a distance of $3.9 \mathrm{kpc}$ to IRAS 18511 (Molinari et al. 1996). Ishii et al. (2002) detected the $3 \mu \mathrm{m}$ water ice feature towards the central object suggesting a visual extinction of 22 mag along this line of sight. Zhang et al. (2005) have reported strong indications of an outflow in IRAS 18511 using the ${ }^{12} \mathrm{CO}$ line. According to them, the mass-loss rate is $\sim 3.7 \times 10^{-4} M_{\odot} \mathrm{yr}^{-1}$ and the dynamical timescale is $5 \times 10^{4} \mathrm{yr}$. Brand et al. (2001) imaged IRAS 18511 using the IRAM 30-m and KOSMA telescopes in a number of molecular lines $\left({ }^{13} \mathrm{CO}, \mathrm{CS}, \mathrm{HCO}^{+}\right)$and find blue shifted and red shifted components distributed over various clumps.

In this study, we explore in detail the region surrounding the IRAS source $18511+0146$ using infrared, sub-millimetre, and radio continuum data. In Sect. 2, we summarize the observations. In Sect. 3, we present the observational results and in Sect. 4, a model of the relative distributions of gas and stars is examined. A discussion of IRAS 18511 region is presented in Sect. 5, and a short summary of our conclusions is given in Sect. 6 . 


\section{Observations, available data and data reduction}

\subsection{JCMT-SCUBA}

The sub-millimetre observations of IRAS 18511 at 450 and $850 \mu \mathrm{m}$, using the Submillimetre Common User Bolometer Array (SCUBA) of the James Clerk Maxwell Telescope ${ }^{1}$ were carried out on 28 May 1999. The data were processed using their standard pipeline SCUBA User Reduction Facility (SURF). The planet Uranus was used for calibration. Submillimetre maps were generated at 450 and $850 \mu \mathrm{m}$ and the fluxes extracted. The beam size is $10^{\prime \prime}$ at $450 \mu \mathrm{m}$ and $15^{\prime \prime} .5$ at $850 \mu \mathrm{m}$. The sensitivities are $0.3 \mathrm{Jy} /$ beam and $0.04 \mathrm{Jy} /$ beam at 450 and $850 \mu \mathrm{m}$, respectively. The flux densities extracted were used to construct the Spectral Energy Distribution (SED) of IRAS 18511.

\subsection{Spitzer data}

The Spitzer Space Telescope ${ }^{2}$ (Werner et al. 2004) was launched in space in August 2003 and consists of a 0.85-m telescope with three cryogenically cooled instruments: InfraRed Array Camera (IRAC), Multiband Imaging Photometer for Spitzer (MIPS) and InfraRed Spectrograph. We have used data from the Legacy projects, GLIMPSE (PI: E. Churchwell) and MIPSGAL (PI: S. Carey) in this paper. The GLIMPSE and MIPSGAL images have been obtained using the software "Leopard".

\subsubsection{MIPSGAL}

The Multiband Imaging Photometer for Spitzer (MIPS) provides the Spitzer Space Telescope with capabilities for imaging and photometry in broad spectral bands centred nominally at 24,70 , and $160 \mu \mathrm{m}$, and for low-resolution spectroscopy between 55 and $95 \mu \mathrm{m}$ (Rieke et al. 2004). The Multiband Imaging Photometer for Spitzer Galactic Plane Survey (MIPSGAL) surveyed the sky in identical regions as covered by GLIMPSE (next subsection) at 24 and $70 \mu \mathrm{m}$ using the MIPS instrument (Carey et al. 2005). The instrument achieves diffraction-limited resolution of $6^{\prime \prime}$ and $18^{\prime \prime}$ at 24 and $70 \mu \mathrm{m}$, respectively. The pixel size is $22^{\prime \prime} 55$ at $24 \mu \mathrm{m}$ and 9.'98 at $70 \mu \mathrm{m}$. The Spitzer Science Center provides final mosaics called Post-Basic Calibrated Data (PBCD) products. These are the maps of multiple calibrated image frames or BCDs (Basic Calibrated Data). For IRAS 18511, we found that these products satisfied our scientific goals. We have carried out extraction and photometry of the sources in the region around IRAS 18511 using the MIPSGAL PBCD images at $24 \mu \mathrm{m}$ and $70 \mu \mathrm{m}$ (details given in Appendix A). The details of the extracted sources and their fluxes are presented in Sect. 3.

\subsubsection{GLIMPSE}

In the GLIMPSE (Galactic Legacy Infrared Midplane Survey Extraordinaire; Benjamin et al. 2003) project, the Spitzer Space Telescope surveyed approximately 220 square degrees of the Galactic plane covering a latitude range of $|b|<1^{\circ}$ and a longitude range of $10^{\circ} \leq l \leq 65^{\circ},-65^{\circ} \leq l \leq-10^{\circ}$. This survey

1 This paper makes use of data from the James Clerk Maxwell Telescope (JCMT) Archive. The JCMT is operated by the Joint Astronomy Centre on behalf of the UK Particle Physics and Astronomy Research Council, the National Research Council of Canada and the Netherlands Organisation for Pure Research.

2 This work is based in part on observations made with the Spitzer Space Telescope, which is operated by the Jet Propulsion Laboratory, under NASA contract 1407. is carried out in the 4 IRAC bands. IRAC is a four-channel camera that provides simultaneous $5.2 \times 5.2$ images at 3.6, 4.5, 5.8, and $8.0 \mu \mathrm{m}$ with a pixel size of $11^{\prime \prime} 2 \times 1$ '.' 2 (Fazio et al. 2004). The resolutions achieved by IRAC are 2.' 4,2 .' 4,2 .' 8 and 3 .' 0 in the 3.6, 4.5, 5.8 and $8.0 \mu \mathrm{m}$ bands, respectively.

The sources in the IRAS 18511 region have been extracted from the GLIMPSE More Complete Archive. The GLIMPSE archive contains point sources with peak signal-to-noise ratio greater than 5 in at least one band. The extracted sources have been used in constructing the spectral energy distributions. However, the bright/saturated sources are not extracted in the GLIMPSE-catalog. In order to get a lower limit on the fluxes of these sources, we have carried out aperture photometry of these bright/saturated sources on GLIMPSE-IRAC PBCD images. For these bright sources, we have taken an aperture radius of 10 pixels and a sky annulus of 15 pixels in order to estimate the fluxes. The approximate centre was determined from the radial profiles using the task "imexamine" in Image Reduction and Analysis Facility (IRAF). For such a combination of aperture radius and sky annulus, no aperture correction needs to be applied.

The PBCD images have been used to study the spatial distribution of sources as well as near and mid infrared emission from this region.

\subsection{Palomar data}

Deep near infrared observations of a $\sim 3^{\prime} \times 3^{\prime}$ field surrounding IRAS 18511 were obtained on 26 July 1999 with the Palomar Observatory 60 -inch telescope equipped with the near infrared IRC-NICMOS3 camera. The field was observed in three broad bands: $J, H$, and $K_{\mathrm{s}}$. A standard dithering technique was used to efficiently remove the sky emission and correct for hot or dead pixels in the $256 \times 256$ NICMOS 3 detector. The data were processed using recipes in IRAF to produce the final images. Photometric calibration was achieved by observing a set of standard stars from the lists of Hunt et al. (1998) and Persson et al. (1998). Astrometric calibration was performed tying the observed positions of bright and isolated sources in the Palomar field to the corresponding entries in the Two Micron All-Sky Survey (2MASS) database. We estimate this procedure to be accurate within 0.5 .

The limiting magnitudes of our observations are found to be $18.1,18.0$, and 16.9 in the $J, H$, and $K_{\mathrm{s}}$ bands, respectively.

\subsection{VLA}

We have also analysed the data for IRAS 18511 at 8.5 and $15 \mathrm{GHz}$ taken from the NRAO Data Archive (project ID: AD406) obtained using the Very Large Array (VLA) in the D configuration. The observation was carried out in Dec. 1997 by Watt \& Mundy (1999). The total on-source integration time in each band is $30 \mathrm{~min}$. The flux calibrators were $1331+305$ and $0137+331.1832-105$ was used as the phase calibrator. The NRAO Astronomical Image Processing System (AIPS) was used for reduction of the data. The beam sizes are 8 ". $5 \times 77^{\prime \prime} .2$ and $5 . \prime 2 \times 4 . .4$ at 8.5 and $15 \mathrm{GHz}$, respectively. The rms noise in the maps are $0.05 \mathrm{mJy} /$ beam and $0.13 \mathrm{mJy} / \mathrm{beam}$ at 8.5 and $15 \mathrm{GHz}$, respectively. 

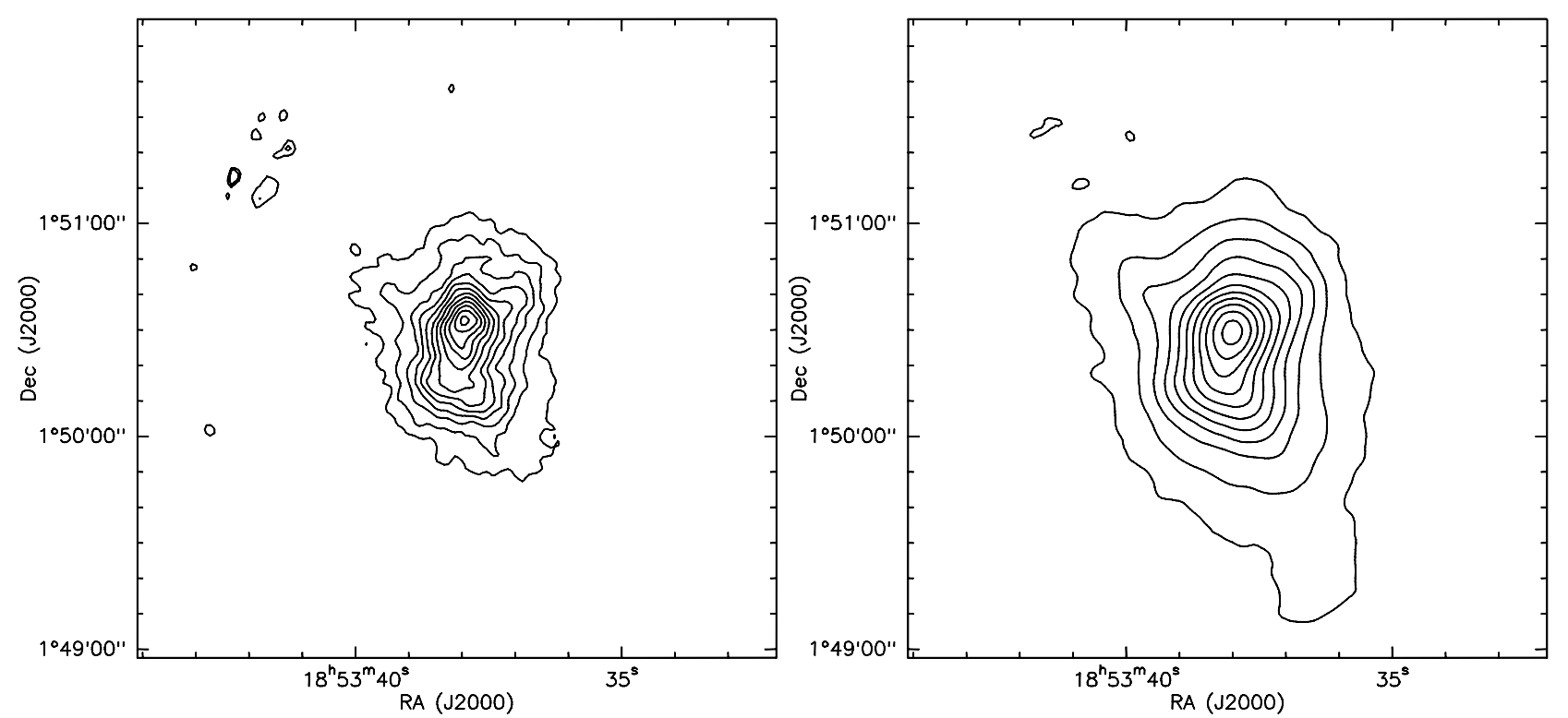

Fig. 1. (Left) JCMT-SCUBA $450 \mu \mathrm{m}$ emission from IRAS 18511 region. The contours levels are from 2.0 to $13.4 \mathrm{Jy} / \mathrm{beam}$ (peak flux density 13.4 Jy/beam) in steps of $1 \mathrm{Jy} / \mathrm{beam}$, where the beam size is $10^{\prime \prime}$. (Right) JCMT-SCUBA $850 \mu \mathrm{m}$ emission from IRAS 18511 region. The contours levels are from 0.18 to $2.28 \mathrm{Jy} /$ beam (peak flux density $\sim 2.28 \mathrm{Jy} /$ beam) in steps of $0.2 \mathrm{Jy} /$ beam, beam is $15^{\prime \prime} 5$.

\section{Results}

\subsection{Submillimetre emission from cold dust}

The JCMT-SCUBA maps at 450 and $850 \mu \mathrm{m}$ trace the emission from cold dust in the region of IRAS 18511. The JCMT maps are shown in Fig. 1. The integrated flux densities upto $10 \%$ contour levels are $139 \mathrm{Jy}$ and $14 \mathrm{Jy}$ at 450 and $850 \mu \mathrm{m}$, respectively. The peak flux densities are $13.4 \mathrm{Jy} /$ beam and $2.28 \mathrm{Jy} /$ beam at 450 and $850 \mu \mathrm{m}$, respectively. The half-power sizes are $0.4 \times 0.6 \mathrm{pc}^{2}$ at $450 \mu \mathrm{m}$ and $0.5 \times 0.7 \mathrm{pc}^{2}$ at $850 \mu \mathrm{m}$. The emission maps show the presence of a core $\left(\alpha_{J 2000}, \delta_{J 2000} \sim 18^{\mathrm{h}} 53^{\mathrm{m}} 38^{\mathrm{s}} .00,+01^{\circ} 50^{\prime} 29^{\prime \prime} .1\right)$ as well as extended emission, particularly to the south. The cold dust emission shows an extension towards the south-east $\left(\alpha_{J 2000}\right.$, $\left.\delta_{J 2000} \sim 18^{\mathrm{h}} 53^{\mathrm{m}} 38^{\mathrm{s}} .13,+01^{\circ} 50^{\prime} 21^{\prime \prime} .6\right)$ which veers towards west further south. Emission towards the north-east of the core is also discerned.

The emission at $450 \mu \mathrm{m}$ as well as at $850 \mu \mathrm{m}$ can be used to estimate the dust temperature of the core as follows. We know that the flux density, $F_{v}$, for optically thin emission can be written as

$F_{v}=\Omega B_{v}\left(T_{\mathrm{d}}\right) \tau_{v}$

where $\Omega$ is the beam solid angle, $B_{v}$ is the Planck function, $\tau_{v}$ is the optical depth at frequency $v$ and $T_{\mathrm{d}}$ is the dust temperature. Assuming, $\tau_{v} \propto v^{\beta}$, the ratio of flux densities at any two wavelengths is a function of $T_{\mathrm{d}}$ and $\beta$. We have assumed $\beta=2$ and used the ratio of fluxes (after convolving the $450 \mu \mathrm{m}$ map to the beam resolution of $15^{\prime \prime} .5$ corresponding to the $850 \mu \mathrm{m}$ beam) in order to obtain a dust temperature of $37 \pm 5 \mathrm{~K}$ at the peak of the emission. The total mass of the cloud has been estimated using the formalism of Hildebrand (1983). Assuming an average dust temperature of $20-30 \mathrm{~K}$ for the entire cloud and using the value of dust emissivity at $850 \mu \mathrm{m}\left(\kappa_{850 \mu \mathrm{m}}=1.5 \mathrm{~cm}^{2} \mathrm{~g}^{-1}\right)$ appropriate to dark clouds from Bianchi et al. (2003), the total mass of the cloud is estimated to be $\sim 750-1310 M_{\odot}$. This mass estimate can be compared with the gas mass of $826 M_{\odot}$ obtained by Watt \& Mundy (1999) using the $\mathrm{C}^{18} \mathrm{O}$ measurements. Assuming $750 M_{\odot}$ to be distributed in a spherical volume of radius $0.6 \mathrm{pc}$, we find the mean density to be $3.4 \times 10^{4} \mathrm{~cm}^{-3}$. For this mass and mean density, the free fall time of the cloud is estimated to be $\sim 3 \times 10^{5}$ yrs.

The visual extinction (in magnitudes) for constant temperature and dust density distribution along the line-of-sight can be obtained by using the following expression:

$I_{v}=\frac{\kappa_{v}}{\kappa_{\mathrm{V}}} \times \frac{A_{V}}{1.086} \times B_{v}(T)$

where $I_{v}$ is the flux density along the line-of-sight, $\kappa_{v} / \kappa_{V}$ is the dust emissivity normalised to emissivity in the $V$-band, and $A_{V}$ is the extinction along the line-of-sight. For a dust temperature of $37 \pm 5 \mathrm{~K}$ at IRAS 18511 core and $\kappa_{850} \mu \mathrm{m} / \kappa_{V}=4.0 \times 10^{-5}$ obtained by Bianchi et al. (2003) for dark clouds, the maximum extinction using the peak $850 \mu \mathrm{m}$ flux is estimated to be $86 \pm 15$ mag. The average extinction has been estimated assuming that the total mass is distributed uniformly in the area enclosed within the $10 \%$ contour level $\left(\sim 1.2 \times 1.8 \mathrm{pc}^{2}\right)$ at $850 \mu \mathrm{m}$. Assuming a temperature of $20-30 \mathrm{~K}$ over this region, the average value of extinction is estimated to be $A_{V} \sim 42-24 \mathrm{mag}$.

\subsection{Mid infrared emission}

The MIPSGAL $70 \mu \mathrm{m}$ image shows the presence of three sources in this region. This image is shown in Fig. 2. The IRAS position is also marked. These sources are better resolved in the MIPS $24 \mu \mathrm{m}$ image. For convenience, we call these sources A, B and C. The location of these sources is shown on the $24 \mu \mathrm{m}$ image in Fig. 2. While the IRAS peak coinicides with A, B lies nearly $20^{\prime \prime}(0.4 \mathrm{pc})$ to the south-west of A. C is the faintest of the three and is separated by $\sim 90^{\prime \prime}$ from A. A is saturated in the MIPS $24 \mu \mathrm{m}$ image. The fluxes and positions of these sources have been extracted using the methods described in the Sect. 2.2.1. Table 1 lists the positions as well as fluxes of A, B and C, respectively.

The four GLIMPSE-IRAC images show a bright source at A and a small compact group of stars located to the south-west of this source (associated with B). Figure 2 shows the locations of $\mathrm{A}, \mathrm{B}$ and $\mathrm{C}$ on the grayscale $8.0 \mu \mathrm{m}$ image. The IRAC $3.6 \mu \mathrm{m}$ image of IRAS 18511 is also shown in Fig. 2. The bright source 

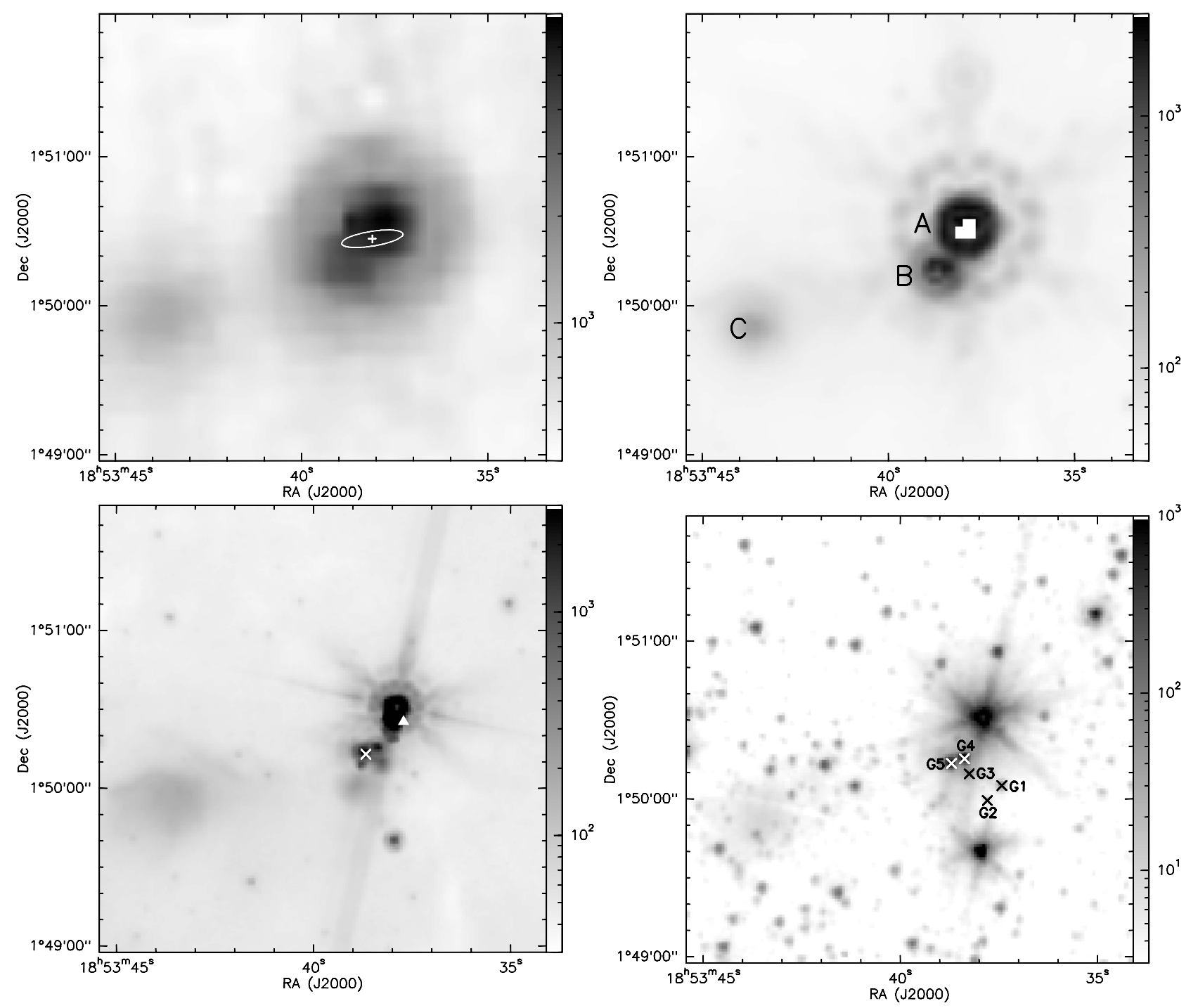

Fig. 2. (Top left) MIPS $70 \mu \mathrm{m}$ image of IRAS 18511 region. The plus symbol and the ellipse represent the IRAS position and the corresponding 1- $\sigma$ position error ellipse. (Top right) MIPS $24 \mu \mathrm{m}$ image of IRAS 18511 region with the sources A, B and C marked. (Bottom left) IRAC $8.0 \mu \mathrm{m}$ image of IRAS 18511 region. The white cross marks the position of radio emission at 8.5 and $15 \mathrm{GHz}$. The solid triangle marks the position of the methanol maser. (Bottom right) IRAC $3.6 \mu$ m image with sources G1-G5 marked.

Table 1. The MIPS flux density details of sources in IRAS 18511.

\begin{tabular}{cccc}
\hline \hline Source & Position & \multicolumn{2}{c}{ Flux Density $(\mathrm{Jy})$} \\
\hline & J2000 & $24 \mu \mathrm{m}$ & $70 \mu \mathrm{m}$ \\
\hline A & $18^{\mathrm{h}} 53^{\mathrm{m}} 37.85+01^{\mathrm{d}} 50^{\prime} 30^{\prime} 5$ & $>20.6$ & 145.7 \\
B & $18^{\mathrm{h}} 53^{\mathrm{m}} 38^{\mathrm{s}} .44+01^{\mathrm{d}} 50^{\prime} 15^{\prime \prime} 1$ & 6.4 & 61.2 \\
C & $18^{\mathrm{h}} 53^{\mathrm{m}} 43.55+01^{\mathrm{d}} 49^{\prime} 500^{\prime} 4$ & 0.8 & 22 \\
\hline
\end{tabular}

associated with A is saturated in all the four IRAC bands. However we have extracted the lower limits to the flux of this source using the method of aperture photometry as mentioned in Sect. 2.2.2. A comparison of the MIPSGAL $24 \mu \mathrm{m}$ image with the higher angular resolution IRAC image shows that B consists of a group of sources. One can see an extension corresponding to $\mathrm{A}$ in the $8.0 \mu \mathrm{m}$ image suggesting that $\mathrm{A}$ may consist of two or more sources. In the GLIMPSE 3.6 or $4.5 \mu \mathrm{m}$ images, the emission from $\mathrm{C}$ is very faint. The emission from $\mathrm{C}$ is diffuse in appearance and stronger in the $8.0 \mu \mathrm{m}$ image.

\subsubsection{The filaments around IRAS 18511}

An investigation into the $8.0 \mu \mathrm{m}$ image shows diffuse emission in the neighbourhood of IRAS 18511. It is particularly interesting to note the filamentary structures (white in Fig. 3) seen in absorption against this diffuse emission. IRAS 18511 seems to lie on one clump and extension of this clump (in the form of filaments) can be seen towards the south-west of A. A clumpy structure can be observed towards the east of IRAS 18511. An estimate of extinction towards these filamentary and clumpy structures has been obtained by using the following relation:

$I=I_{0} \mathrm{e}^{-\tau_{8}}$

where $I_{0}$ is the background source intensity (determined in the vicinity of the filamentary structures), $I$ is the observed intensity, and $\tau_{8}$ is the optical depth at $8.0 \mu \mathrm{m}$. One observes considerable variations of $I_{0}$ across the image. We have therefore assumed a different value of $I_{0}$ for each pixel as follows. A median filter image has been constructed using a window size of 99 pixels $\left(2^{\prime}\right)$. Using the median value at every pixel as the corresponding $I_{0}$, a visual extinction $\left(A_{\mathrm{V}}\right)$ map has been constructed 


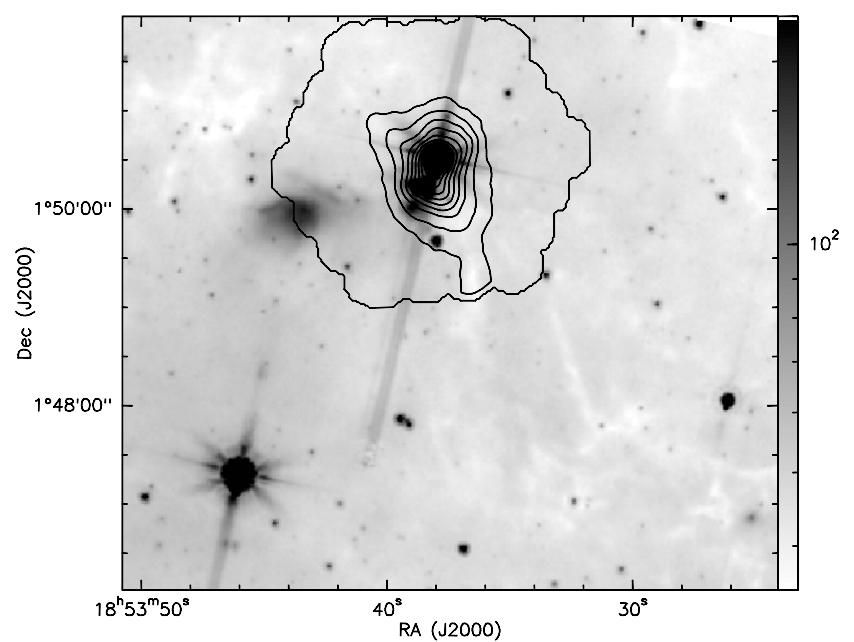

Fig. 3. Grayscale image of IRAS 18511 at GLIMPSE-IRAC $8.0 \mu \mathrm{m}$ showing the dark filaments against diffuse emission. Overlaid as contours is the JCMT-SCUBA sub-millimetre emission at $850 \mu \mathrm{m}$. The region covered by the SCUBA map is also shown.

taking $A_{\mathrm{V}} / \tau_{8}=22.5$. This relation $\left(A_{\mathrm{V}} / \tau_{8}\right)$ has been obtained by taking the ratios of extinctions $\left(A_{8} / A_{K}=0.43, A_{K} / A_{\mathrm{V}}=0.112\right)$ obtained by Indebetouw et al. (2005) and Rieke \& Lebofsky (1985). The pixels representing values higher than the median have been masked. The $A_{V}$ values are found to have values in the range 6-8 in the filaments as well as towards the clumpy structures. Converting the $A_{\mathrm{V}}$ map to a column density map, the mass in the filaments as well as in the clumps have been estimated. While the mass of gas in the clump $\left(\alpha_{J 2000}, \delta_{J 2000} \sim\right.$ $\left.18^{\mathrm{h}} 53^{\mathrm{m}} 47^{\mathrm{s}} .3+01^{\circ} 51^{\prime} 0^{\prime \prime}\right)$ is found to be $\sim 46 M_{\odot}$, the mass in a few filaments is found to be in the range $\sim 5-15 M_{\odot}$. The total mass of the filaments and clumpy structures shown in Fig. 3 is $\sim 120 M_{\odot}$. These mass estimates represent lower limits as $I_{0}$ has contributions from foreground as well as background emission. In addition, the total mass estimate also suffers from blanking of pixels near the $8.0 \mu \mathrm{m}$ sources. In particular, since IRAS $18511 \mathrm{~A}$ is very bright at $8.0 \mu \mathrm{m}$, the clump containing IRAS $18511 \mathrm{~A}$ is masked out in the extinction map. It is interesting to note that there is an overlap of a filament in the $8.0 \mu \mathrm{m}$ image with the JCMT-SCUBA $850 \mu \mathrm{m}$ sub-millimetre emission towards the south-west.

\subsubsection{Associated young stellar objects (IRAC colour-colour diagram)}

In order to select IRAC sources from the GLIMPSE catalog for further study, we have defined a "region of interest" around IRAS 18511 . We take this to be a region overlapping the submillimetre emission from cold dust. In other words, we take the sources in a region enclosed by the $10 \%$ contour level of the peak of the the sub-millimetre $850 \mu \mathrm{m}$ emission. We find a total of 39 sources (including the saturated source at A). Of these, six are detected in all four IRAC bands. These have been plotted in the IRAC colour-colour diagram ([3.6]-[4.5] vs. [5.8]-[8.0]) which is shown in Fig. 4. In the diagram, the solid square approximately delineates the region occupied by class II sources whereas the dotted square covers the region occupied by the class I models of Allen et al. (2004) (see their Fig. 4). From the colour-colour diagram in Fig. 4, we find five sources lying within the boxes representing the region occupied by either class I or class II sources. We label these sources G1, G2, G3,

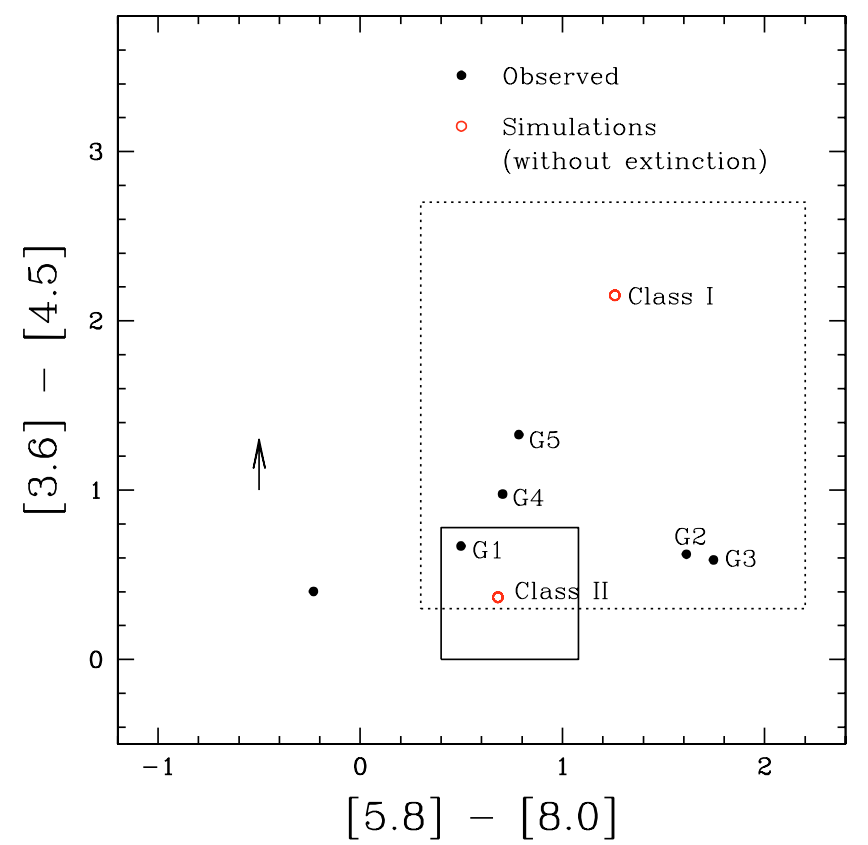

Fig. 4. Spitzer colour-colour diagram of the observed sources and the models used in the simulations. The filled circles represent the sources detected in all four IRAC bands. The empty (red) circles represent the colours of the unreddened class I and class II models used in the simulations (see text for details). The solid line square approximately delineates the region occupied by class II sources whereas the dotted-line square covers the region occupied by the class I models shown in the models of Allen et al. (2004). The arrow represents the extinction vector for $A_{V}=20$ mag.

G4 and G5. Of these 5 objects, G2, G3, G4 and G5 lie within the class I box while G1 lies in the overlap region between class I and class II. These sources are also shown in Fig. 2 and the details of the coordinates and fluxes of these sources are included in Table 2.

\subsection{Near infrared emission}

The near infrared Palomar $K_{\mathrm{s}}$ band image of IRAS 18511 is shown in Fig. 5. Unlike many other star forming regions, we do not detect near infrared nebulosity in these images. Among the sources extracted from the Palomar images in the $J, H$ and $K_{\mathrm{s}}$ bands, we select a sample of sources lying within the "region of interest" (see Sect. 3.2.2 for details). We find a total of 68 sources, including the saturated sources. The fluxes of three sources which are saturated in the Palomar images have been taken from the $2 \mathrm{MASS}^{3}$. This includes the source associated with A, which is saturated in the $H$ and $K_{\mathrm{s}}$ bands of the Palomar images. Of the total of 68 sources, we find that 27 sources are detected in all three $J H K_{\mathrm{s}}$ bands.

\subsubsection{Associated young stellar objects (NIR and IRAC colour-colour diagrams)}

In order to identify the young stellar objects in this region, we have constructed the colour-colour diagram of $J-H$ vs. $H-K_{\mathrm{s}}$

\footnotetext{
3 This publication makes use of data products from the Two Micron All Sky Survey, which is a joint project of the University of Massachusetts and the Infrared Processing and Analysis Center/California Institute of Technology, funded by the NASA and the NSF.
} 
Table 2. Young stellar objects around IRAS 18511 selected from Palomar (NIR) as well as Spitzer-IRAC (MIR) colour-colour diagrams.

\begin{tabular}{|c|c|c|c|c|c|c|c|c|c|c|}
\hline S. No. & $\begin{array}{l}\alpha_{2000} \\
(\mathrm{deg})\end{array}$ & $\begin{array}{l}\delta_{2000} \\
(\mathrm{deg})\end{array}$ & $\begin{array}{c}J \\
(\mathrm{mag})\end{array}$ & $\begin{array}{c}H \\
(\mathrm{mag})\end{array}$ & $\begin{array}{c}K_{\mathrm{s}} \\
(\mathrm{mag})\end{array}$ & $\begin{array}{c}\text { Spitzer-GLIMPSE } \\
\text { designation }\end{array}$ & $\begin{array}{l}3.6 \mu \mathrm{m} \\
(\mathrm{mag})\end{array}$ & $\begin{array}{l}4.5 \mu \mathrm{m} \\
(\mathrm{mag})\end{array}$ & $\begin{array}{l}5.8 \mu \mathrm{m} \\
(\mathrm{mag})\end{array}$ & $\begin{array}{l}8.0 \mu \mathrm{m} \\
(\mathrm{mag})\end{array}$ \\
\hline 1 & 283.400479 & 1.841550 & $18.36 \pm 0.20$ & $17.22 \pm 0.13$ & $16.50 \pm 0.19$ & - & - & - & - & - \\
\hline 2 & 283.403321 & 1.840394 & $18.86 \pm 0.36$ & $17.58 \pm 0.21$ & $15.73 \pm 0.14$ & - & - & - & - & - \\
\hline 3 (G1) & 283.405912 & 1.834686 & - & $16.45 \pm 0.09$ & $13.43 \pm 0.02$ & G034.8140+00.3504 & $10.91 \pm 0.11$ & $10.24 \pm 0.11$ & $9.80 \pm 0.32$ & $9.30 \pm 0.10$ \\
\hline 4 & 283.405946 & 1.840603 & $11.85 \pm 0.01$ & $11.58 \pm 0.01$ & $11.11 \pm 0.01$ & - & - & - & - & - \\
\hline 5 & 283.406588 & 1.840244 & $13.12 \pm 0.01$ & $12.67 \pm 0.01$ & $11.89 \pm 0.01$ & - & - & - & - & - \\
\hline $6(\mathrm{G} 2)$ & 283.407446 & 1.833108 & $18.64 \pm 0.37$ & $15.89 \pm 0.08$ & $14.88 \pm 0.07$ & G034.8133+00.3483 & $13.19 \pm 0.11$ & $12.57 \pm 0.14$ & $11.18 \pm 0.24$ & $9.57 \pm 0.10$ \\
\hline $7^{a}$ & 283.407867 & 1.841829 & $13.43 \pm 0.04$ & $9.30 \pm 0.03$ & $6.61 \pm 0.03$ & - & - & - & - & - \\
\hline 8 (G3) & 283.409387 & 1.835869 & - & - & $14.67 \pm 0.08$ & G034.8166+00.3479 & $11.00 \pm 0.21$ & $10.41 \pm 0.21$ & $7.84 \pm 0.16$ & $6.09 \pm 0.27$ \\
\hline 9 & 283.409779 & 1.842842 & $15.95 \pm 0.04$ & $15.00 \pm 0.03$ & $13.38 \pm 0.02$ & - & - & - & - & - \\
\hline $10(\mathrm{G} 4)$ & 283.409896 & 1.837536 & - & $15.00 \pm 0.03$ & $11.11 \pm 0.01$ & G034.8183+00.3482 & $7.56 \pm 0.08$ & $6.58 \pm 0.19$ & $5.65 \pm 0.05$ & $4.95 \pm 0.07$ \\
\hline 11 (G5) & 283.411304 & 1.837044 & - & $17.54 \pm 0.23$ & $12.77 \pm 0.02$ & G034.8185+00.3467 & $7.61 \pm 0.05$ & $6.28 \pm 0.20$ & $5.11 \pm 0.04$ & $4.32 \pm 0.04$ \\
\hline 12 & 283.411446 & 1.832517 & $17.69 \pm 0.15$ & $17.21 \pm 0.21$ & $16.64 \pm 0.26$ & - & - & - & - & - \\
\hline 13 & 283.412083 & 1.833464 & $17.19 \pm 0.09$ & $15.55 \pm 0.04$ & $14.16 \pm 0.03$ & - & - & - & - & - \\
\hline 14 & 283.414129 & 1.839558 & $17.88 \pm 0.14$ & $17.31 \pm 0.14$ & $16.86 \pm 0.32$ & - & - & - & - & - \\
\hline 15 & 283.415887 & 1.835761 & $14.53 \pm 0.02$ & $13.93 \pm 0.02$ & $13.66 \pm 0.02$ & G034.8194+00.3420 & $13.42 \pm 0.17$ & $13.20 \pm 0.13$ & - & - \\
\hline 16 & 283.415892 & 1.834844 & $18.02 \pm 0.16$ & $16.28 \pm 0.06$ & $14.66 \pm 0.04$ & G034.8187+00.3417 & $13.15 \pm 0.14$ & $12.81 \pm 0.12$ & - & - \\
\hline
\end{tabular}

${ }^{a}$ Source saturated in the GLIMPSE-IRAC images.

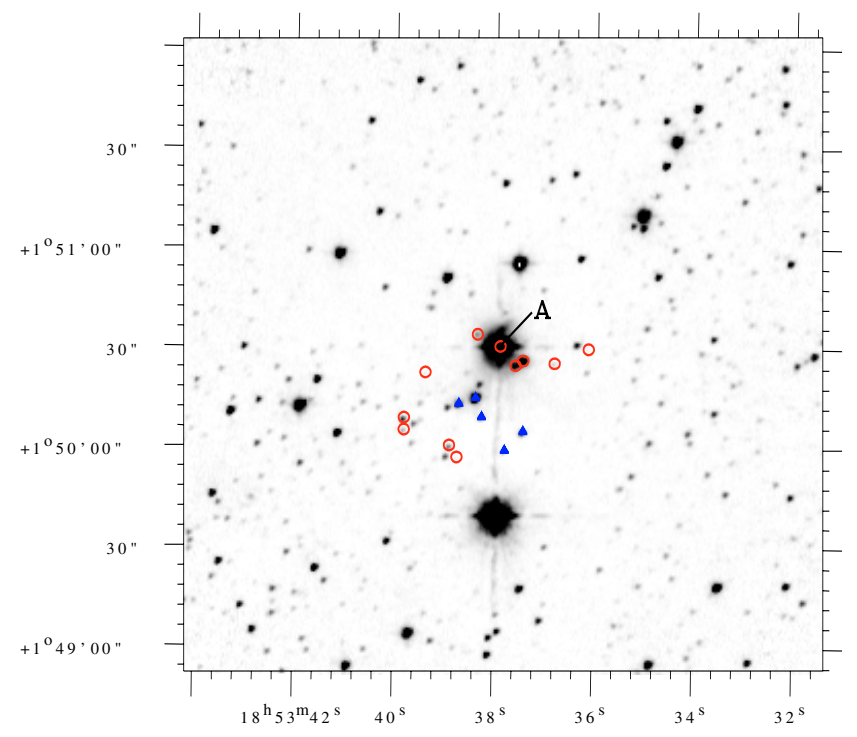

Fig. 5. $K_{\mathrm{S}}$ band Palomar image of the region around IRAS 18511. The axes are in J2000 coordinates. The sources marked by open (red) circles are the YSO candidates selected from the various colour-colour diagrams (see Table 2). The (blue) solid triangles are the sources G1-G5.

using the 27 sources detected in $J H K_{\mathrm{s}}$ bands. This is shown in Fig. 6 (left). The loci of the main-sequence and giant branches are shown by the solid and dotted lines, respectively. While the short-dashed line represents the locus of T Tauri stars (Meyer et al. 1997), the dot-dashed line represents the reddening vector of the main-sequence stars. The long-dashed line shows the locus of Herbig Ae/Be stars (Lada \& Adams 1992). We have assumed extinction values of $A_{J} / A_{V}=0.282, A_{H} / A_{V}=0.175$ and $A_{K} / A_{V}=0.112$ from Rieke \& Lebofsky (1985). The sources having infrared excess are those lying to the right of the reddening curve. We find a total of 10 sources having infrared-excess. These sources are included in Table 2. The source associated with IRAS $18511 \mathrm{~A}$ has an infrared excess as well as very large reddening $\left(J-H \sim 4.1, H-K_{\mathrm{s}} \sim 2.7\right)$ as can be seen in the CC diagram. IRAS $18511 \mathrm{~A}$ is listed as source number 7 in Table 2.
We have also searched for Spitzer-IRAC counterparts to the near infrared objects. We have used a search radius of 0.8 . A total of 24 Palomar sources have IRAC counterparts. Wang \& Looney (2007) have used $J-[3.6]$ vs. $K_{\mathrm{s}}-$ [4.5] colour-colour diagram to identify the young stellar objects in groups around Herbig Ae/Be stars. In our sample we find 6 objects that are detected in the $J, K_{\mathrm{s}}$, IRAC1 $(3.6 \mu \mathrm{m})$ as well as IRAC2 $(4.5 \mu \mathrm{m})$ bands. These objects have been plotted in the $J-[3.6]$ vs. $K_{\mathrm{s}}-[4.5]$ colour-colour diagram in Fig. 6 (right). All the sources in this colour-colour diagram have been dereddened by 7 mag of visual extinction $\left(1.8 \mathrm{mag} \mathrm{kpc}^{-1}\right)$, corresponding to the extinction due to interstellar medium (Whittet 1992). We have used the Wang \& Looney (2007) line (their Eq. (1)) to separate the normal stars and the young stellar objects. This is shown by the solid line in the Fig. 6 . The dotted line represents the young stellar object (YSO) locus given by them. From this diagram, we find 3 objects which can be characterised as YSO candidates based on the above criterion. The details of the 3 YSO candidates from the $J-[3.6]$ vs. $K_{\mathrm{s}}-[4.5]$ are included in Table 2. All the young stellar objects selected from various colour-colour diagrams ([3.6] - [4.5] vs. [5.8] - [8.0], $J-H$ vs. $H-K_{\mathrm{s}}, J-[3.6]$ vs. $\left.K_{\mathrm{s}}-[4.5]\right)$ are overplotted on the grayscale Palomar $K_{\mathrm{s}}$ band image in Fig. 5 and listed in Table 2.

\subsection{Radio emission from ionised gas}

The VLA data show weak emission (point source) at both 8.5 and $15 \mathrm{GHz}$ in the IRAS 18511 region. The emission is from a point source with coordinates $\left(\alpha_{J 2000}, \delta_{J 2000}=\right) 18^{\mathrm{h}} 53^{\mathrm{m}} 38^{\mathrm{s}} .67+$ $01^{\circ} 50^{\prime} 13^{\prime \prime} .0$. The location of the radio point source coincides with G5, which is marked as a cross in the $8.0 \mu \mathrm{m}$ image in Fig. 2. The flux densities are $0.69 \mathrm{mJy} / \mathrm{beam}$ and $0.68 \mathrm{mJy} / \mathrm{beam}$ at 8.5 and $15 \mathrm{GHz}$, respectively. The size of the radio emitting region is $\lesssim 5^{\prime \prime}$ which corresponds to $0.09 \mathrm{pc}$ at the distance of IRAS 18511 . The spectral index is $-0.03_{-0.30}^{+0.23}$ indicating the nature of this emission to be (optically thin) free-free emission. Thus, the ionised gas here is from a small extremely compact region around this source. Using the formulation of Schraml \& Mezger (1969), as well as Panagia (1973), the ZAMS spectral type of this source is estimated to be B2-B1 (flux of Lyman continuum photons is $\sim 1.3 \times 10^{45} \mathrm{~s}^{-1}$ ). There is no radio 

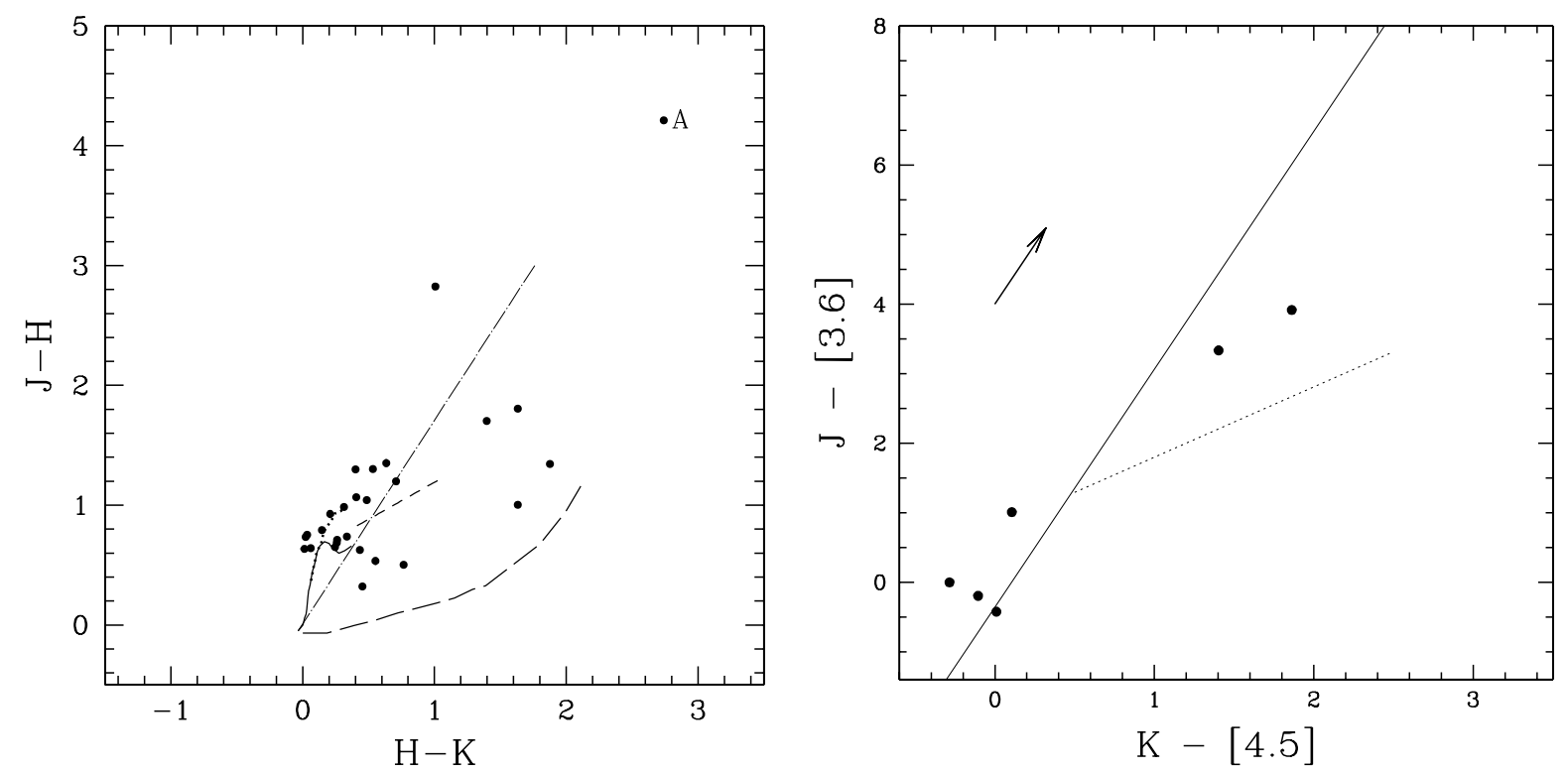

Fig. 6. Colour-colour diagrams (CCDs) of sources detected in Palomar and Spitzer-IRAC images within 10\% contour level of the sub-millimetre $850 \mu \mathrm{m}$ peak at IRAS 18511 A (see text for details). (Left) $J-H$ vs. $H-K_{\mathrm{s}}$ CCD where the locii of the main sequence and giant branches are shown by the solid and dotted lines respectively. The short-dash line represents the locus of T-Tauri stars. The dash-dotted straight line follows the reddening vectors of main sequence stars (or dwarfs). The long dashed line represents the locus of Herbig Ae/Be stars. The position of IRAS 18511 A is marked as "A" in the CCD. (Right) $J-[3.6]$ vs. $K_{\mathrm{s}}-[4.5] \mathrm{CCD}$ where the solid line represents the line separating the normal stars and the young stellar objects while the dotted line represents the YSO locus of Wang \& Looney (2007). The arrow represents the extinction vector $A_{V}=5$ mag.

emission detected from the other sources in this region, including the bright A source, upto $2 \sigma$ levels of $100 \mu \mathrm{Jy}$ and $300 \mu \mathrm{Jy}$ at 8.5 and $15 \mathrm{GHz}$, respectively.

\subsection{Source $C$}

The source $\mathrm{C}$ is clearly detected at 24 and $70 \mu \mathrm{m}$ and appears as a faint diffuse emission. The weak diffuse emission shows filamentary structures within it. Also, towards the north, we discern a filamentary structure with a sharp edge. The cause of this emission is unclear. The emission at $8.0 \mu \mathrm{m}$ is stronger than at $24 \mu \mathrm{m}$ indicating the presence of strong Polycyclic Aromatic Hydrocarbons (PAH) emission. The angular size of $\mathrm{C}$ is $\sim 35^{\prime \prime}$ which corresponds to a size of $0.7 \mathrm{pc}$ at $3.9 \mathrm{kpc}$ (distance to IRAS 18511). While $\mathrm{C}$ being an irregular galaxy cannot be ruled out, it is possible that $\mathrm{C}$ is a Galactic object.

\subsection{Spectral energy distributions}

The fluxes at different wavelengths have been extracted for various sources in IRAS 18511 region and these have been used in constructing their SEDs. For the sake of comparison, we have also constructed the SED of IRAS 18511 as a single source using IRAS as well as MSX fluxes. The luminosity obtained by integrating the area under the IRAS-MSX curve gives an estimate of the total luminosity. Figure 7 (left) shows the SED of IRAS 18511 constructed using IRAS and MSX fluxes. Also plotted in this figure is the SED of A. It is to be noted that the fluxes of $\mathrm{A}$ at the IRAC and MIPS $24 \mu \mathrm{m}$ band are lower limits as A is saturated in these wavebands. The luminosity of IRAS 18511 , obtained by integrating the IRAS-MSX SED, is $1.1 \times 10^{4} \mathrm{~L}_{\odot}$. The lower limit to the luminosity of A is $7.3 \times 10^{3} L_{\odot}$, derived using Spitzer photometry and JCMT-SCUBA data. Hence, most of the luminosity of IRAS 18511 (at least 66\%) is due to the protostar(s) in A.
The SEDs of the individual members (G1-G5) have been constructed using wavelengths at which they are resolved and detected. These SEDs are shown in Fig. 7 (right) for no dereddening applied. The SEDs of these individual objects have been constructed using the Palomar as well as IRAC fluxes. From Fig. 7, we see that all the SEDs rise rapidly with increasing wavelength. In order to compare the SEDs relative to each other, we have normalised them with respect to the fluxes in $H$ band. The SEDs of G1, G2, G4 and G5 have been dereddened by $A_{\mathrm{V}} \sim 7$ mag of visual extinction (due to ISM) as well as by $A_{\mathrm{V}} \sim$ 22 mag derived by Ishii et al. (2002). Figure 8 shows the SEDs relative to each other for a dereddening of $A_{\mathrm{V}} \sim 7 \mathrm{mag}$ (left) and 22 mag (right), respectively. By integrating the area under the curves (near to mid infrared), we have estimated lower-limits to the luminosities $\left(L_{\mathrm{NIR}-8 \mu \mathrm{m}}\right)$ of these objects. These luminosities are listed in Table 3 for the sources G1-G5 for dereddening of 7 and 22 mag of visual extinction. The source G5 appears to be identical to the VLA source discussed in Sect. 3.4 and hence we expect it to be of B1-B2 spectral type with an effective temperature $\sim 20000 \mathrm{~K}$ and a bolometric luminosity $4400 L_{\odot}$.

\subsubsection{Robitaille et al. models}

From Fig. 8, we see that the sources show a rise in spectral energy distributions with an increase in wavelength. Their $L_{\mathrm{NIR}-8} \mu \mathrm{m}$ (listed in Table 3 ) suggest that at least a few of these are massive young objects. In order to get a qualitative estimate of the evolutionary stage of the cluster members, we have fitted the SED of few cluster members with the models of Robitaille et al. (2007) (hereafter RWIW). They have computed a large set of radiation transfer models and obtained the SEDs for a reasonably large parameter space. These SEDs can be fitted to multiwavelength observational data of single sources to constrain the physical parameters and the evolutionary stage. However, it is important to note that the best constraints are obtained if the near, mid and far infrared as well as sub-millimetre data are included. 

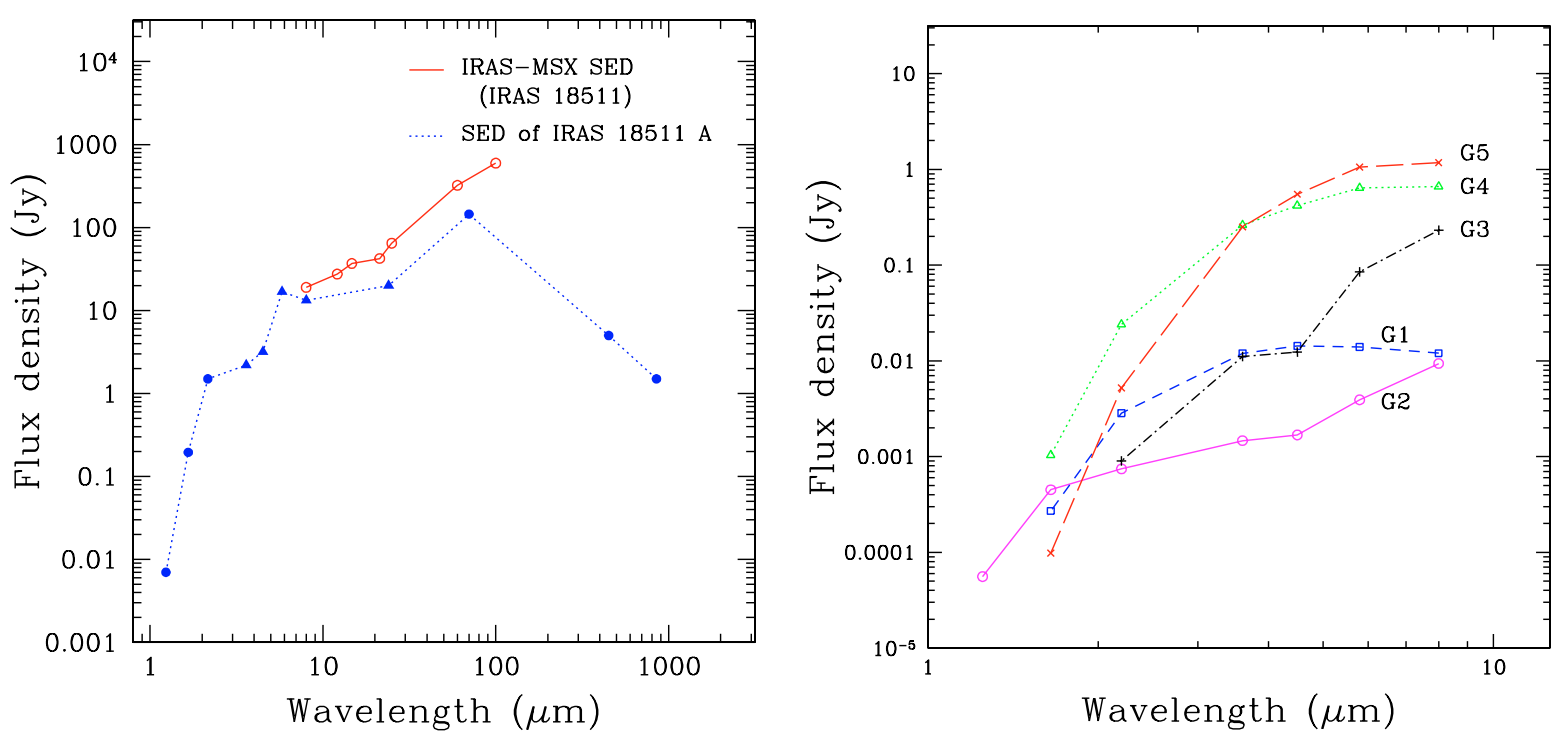

Fig. 7. (Left) The solid line represents the spectral energy distribution (SED) of IRAS 18511 constructed using the IRAS and MSX fluxes. The dotted line depicts the lower limit to the spectral energy distribution of A constructed using Palomar, Spitzer-IRAC, Spitzer-MIPS and JCMTSCUBA. While the filled circles represent the fluxes of IRAS 18511 A, the solid triangles represent the lower limits. (Right) The SEDs of young stellar objects, G1-G5, detected using IRAC colour-colour diagram.
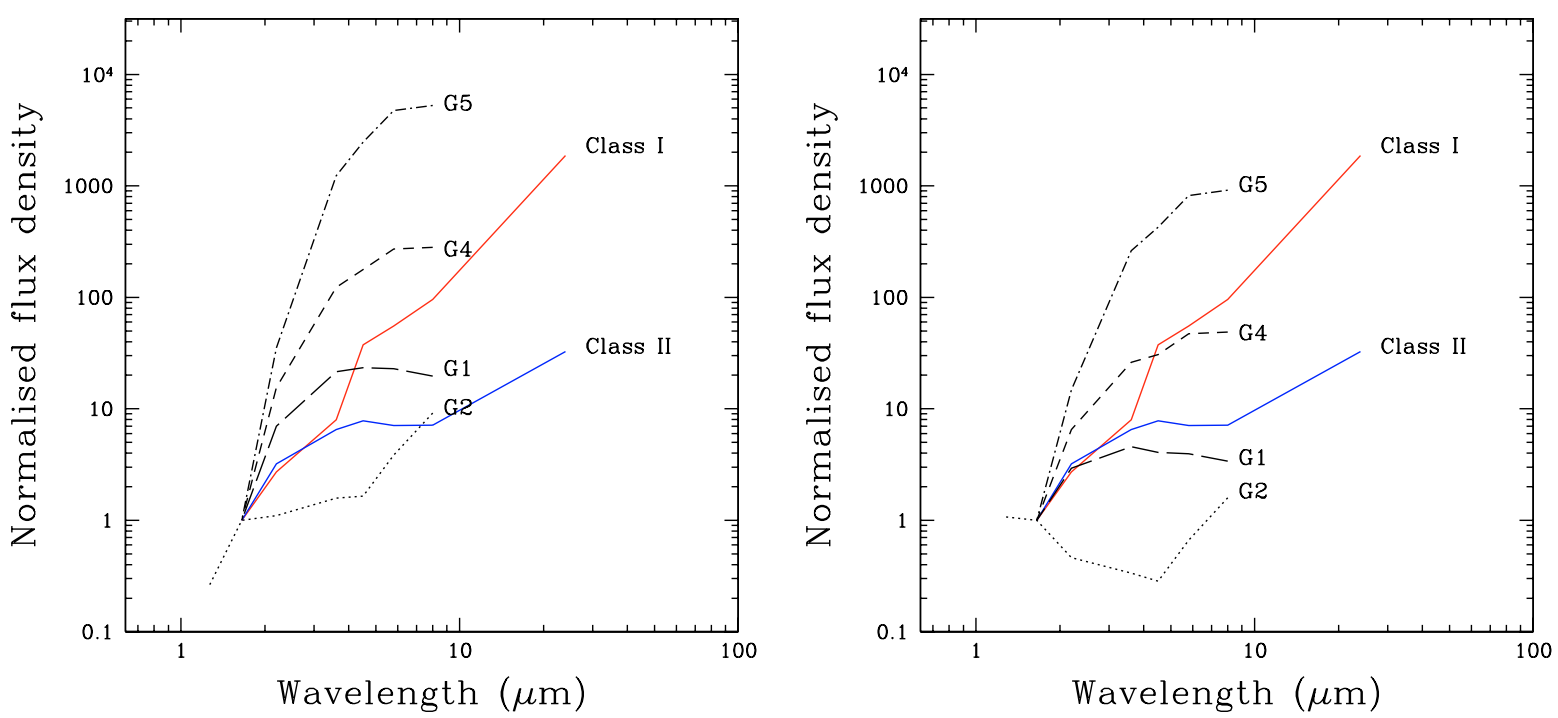

Fig. 8. Normalised spectral energy distribution (with respect to $H$ band) of sources G1, G2, G4 and G5, dereddened by $A_{V} \sim 7$ mag (left) and $A_{V} \sim 22 \mathrm{mag}$ (right). The solid lines represent the normalised spectral energy distributions of the class I (red) and class II (blue) sources adopted in the cluster simulations.

We have fitted the RWIW models to two sources, G4 and G5. The models were fit to the SEDs dereddened by 22 mag of visual extinction. The $L_{\mathrm{NIR}-8 \mu \mathrm{m}} \mathrm{S}$ of G4 and G5 are the highest among the $\mathrm{G}$ sources. The SEDs have been constructed using near infrared Palomar and mid infrared IRAC data. Upper limits at MIPS $24 \mu \mathrm{m}$ (corresponding to the IRAS 18511 B) and at JCMTSCUBA $850 \mu$ m (flux density at the position of IRAS 18511 B) have also been used as constraints to the modelling. It should be noted that our sub-millimetre angular resolution is not sufficient for this purpose and hence the fits are non-unique. We have also put additional constraints based on luminosity and mass of envelope/disk ( $85 M_{\odot}$ based on the sub-millimetre $850 \mu \mathrm{m}$ flux at the position of IRAS $18511 \mathrm{~B}$ ). The models are selected based on the least-squares chi-square method. For G5, additional constraints are available since it is estimated to be of ZAMS spectral type B1-B2 (based on the VLA observations). Among the four models for G5, Model 1 simulates a young protostar and the $T_{\text {eff }}$ of the central object does not produce Lyman continuum photons to ionise the surrounding gas. On the other hand, the other models assume more evolved central stars $\left(T_{\text {eff }} \sim 20000 \mathrm{~K}\right)$. Figure 9 shows some sample RWIW models for G4 and G5 along with the observed SEDs. The parameters are listed in Table 4. In the table, Col. 1 lists the source $(\mathrm{G} 4 / \mathrm{G} 5)$ Col. $2\left(M_{*}\right)$ lists the mass of central object, Col. 3 lists the effective temperature of the central objects, Col. 4 lists the total luminosity, Col. 5 lists the inclination angle with respect to the observer $\left(90^{\circ}\right.$ is edgeon), Col. $6(\dot{M})$ represents the envelope accretion rate, Col. 7 $\left(M_{\text {env }}\right)$ lists the mass of envelope, Col. $8\left(M_{\text {disk }}\right)$ lists the mass of disk, Col. $9\left(A_{\mathrm{V}}\right)$ lists the extinction along the line-of-sight and Col. 10 lists the age. The luminosity listed in the Table is the total (non-isotropic) luminosity (central object + envelope and/or disk) computed by RWIW. 

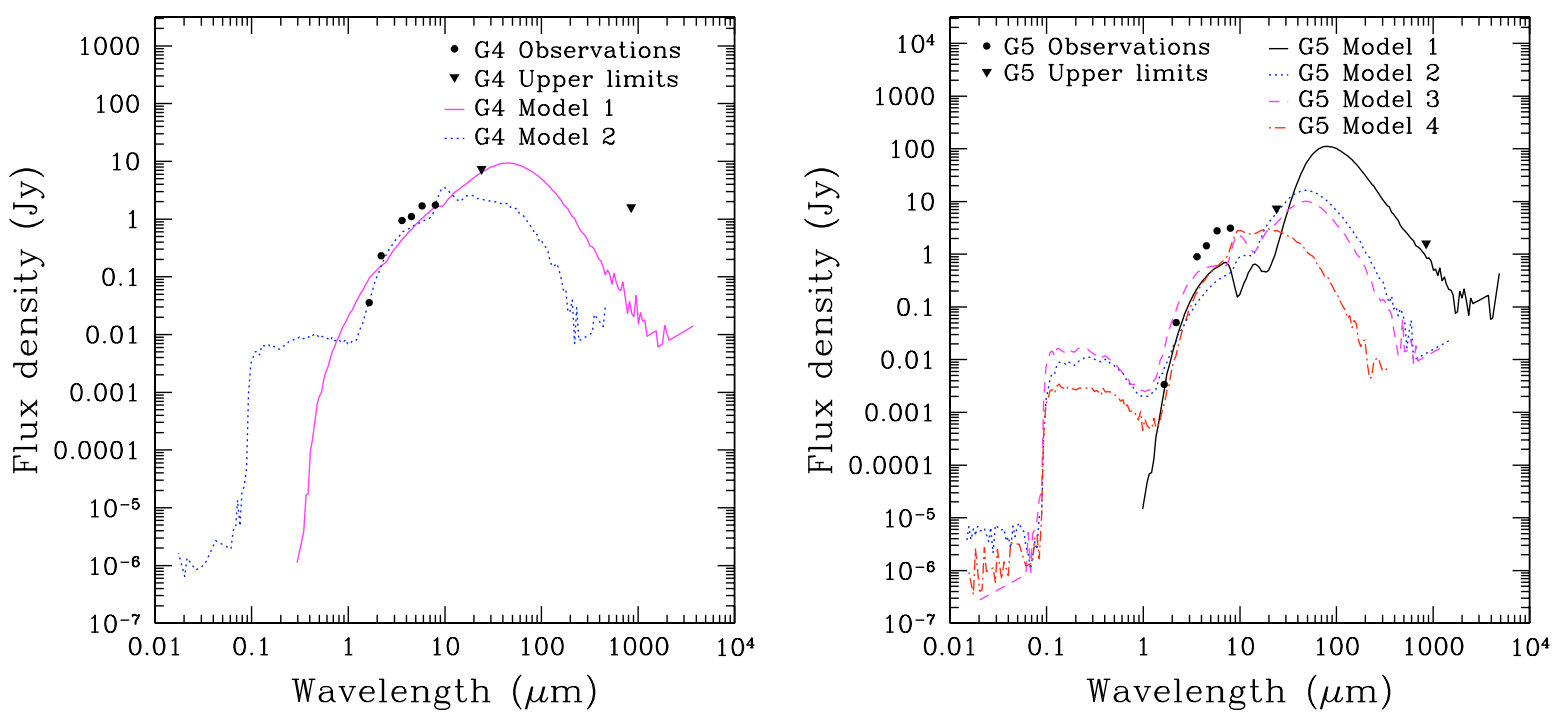

Fig. 9. Spectral energy distributions of G4 (left) and G5 (right) with some sample models of Robitaille et al. (2007) (RWIW). The observed fluxes are shown by filled circles, the triangles denote upper limits to the observed fluxes and the lines represent the RWIW models. The parameters of these models are listed in Table 4.

Table 3. Luminosities of the sources G1-G5 in IRAS 18511 region detected in all four IRAC bands.

\begin{tabular}{ccc}
\hline \hline S. No. & \multicolumn{2}{c}{ Luminosity $\left(L_{\mathrm{NIR}-8 \mu \mathrm{m}}\right)$} \\
& $L_{\odot}\left(A_{V} \sim 7 \mathrm{mag}\right)$ & $L_{\odot}\left(A_{V} \sim 22 \mathrm{mag}\right)$ \\
\hline G1 & 7 & 16 \\
G2 & 2 & 9 \\
G3 & 19 & 36 \\
G4 & 184 & 412 \\
G5 & 238 & 492 \\
\hline
\end{tabular}

It is interesting, especially for G5, that the observed fluxes in the mid infrared (IRAC) bands have a steeper wavelength dependence than predicted by these models which perhaps underestimate the extinction. Another likely possibility is that emission from small grains or from Polycyclic Aromatic Hydrocarbons $(\mathrm{PAH})$ effects the observations. The results indicate that the SEDs of few sources can be explained by massive class I and class II type sources i.e., sources with the massive central objects (7-10 $M_{\odot}$ ) alongwith envelopes/disks. Thus, the models of lowmass objects extended to more massive candidates can reasonably explain the SEDs of some cluster members of IRAS 18511.

\section{Cluster simulations}

In the IRAS 18511 region, a number of sources have been detected in the near and mid infrared. In regions of high extinction like IRAS 18511, these sources could be highly reddened luminous objects or low-luminosity low-extinction objects. The objective of the cluster simulations is to investigate this. We simulate a cluster of young objects embedded in a cloud of gas and ascertain whether the observations of IRAS 18511 in various near and mid infrared bands are in qualitative agreement with the predictions of the model as our statistics are too low for a quantitative comparison. We explore the cluster membership in terms of the fraction of objects of different evolutionary stages (class I and class II). As we are exploring a young embedded cluster, we have not considered objects in the class III phase or later. This modelling will allow us to obtain a qualitative estimate of the evolutionary stage of the cluster by varying the fractions of class I and class II sources in the cluster.

The inputs to these simulations include (a) observables quantities that have been incorporated in the model based on observations, and (b) assumptions. The observables include (1) limit on the most massive object in the cluster, (2) the total luminosity of the cluster, (3) mass and size of the spherical cloud of gas in which the cluster is embedded, and (4) the distance. We have assumed the following: (1) Initial Mass Function (IMF) and (2) Star formation history (SFH). The output that can be compared directly with the observations is the number of detected cluster members in the $K_{\mathrm{s}}(2.12 \mu \mathrm{m})$, IRAC1 $(3.6 \mu \mathrm{m})$, IRAC2 $(4.5 \mu \mathrm{m}), \operatorname{IRAC} 3(5.8 \mu \mathrm{m})$, IRAC4 $(8.0 \mu \mathrm{m})$ and MIPS $1(24 \mu \mathrm{m})$ bands for given completeness limits. A detailed description of the model is given in Appendix B.

\subsection{Results}

\subsubsection{Model}

The upper mass limit of the cluster has been taken to be $10 M_{\odot}$ corresponding to the ZAMS spectral type of a single object inferred from the bolometric luminosity of IRAS 18511. The model has been run for the Salpeter IMF. The mass and radius of the spherical cloud of gas have been taken to be $750 M_{\odot}$ and $0.6 \mathrm{pc}$, respectively. These have been derived from the $850 \mu \mathrm{m}$ JCMT-SCUBA observations. This corresponds to a maximum visual extinction of 69 mag (including the $A_{\mathrm{V}} \sim$ 7 mag due to the ISM). The total luminosity has been taken to be $1.1 \times 10^{4} L_{\odot}$ which is the total luminosity of IRAS 18511 . The model was run 1000 times for each of the following cases of star formation history:

1. Coeval formation with an age of $0.5 \mathrm{Myr}$ corresponding to class I type sources - Every cluster member is assumed to have a class I type spectrum.

2. Coeval formation with an age of $1 \mathrm{Myr}$ corresponding to class II type sources - Every cluster member is assumed to have a class II type spectrum.

3. Uniform star formation rate between $0.01-1 \mathrm{Myr}-\mathrm{This}$ corresponds to the general case incorporating class I as well as 
Table 4. Parameters of the Robitaille et al. (2007) models for G4 and G5, plotted in Fig. 9. Column (2) ( $\left.M_{*}\right)$ lists the mass of central object, Col. (3) lists the effective temperature of the central objects, Col. (4) lists the total luminosity, Col. (5) lists the inclination angle with respect to the observer ( $90^{\circ}$ is edge-on), Col. (6) $(\dot{M})$ represents the envelope accretion rate, Col. (7) $\left(M_{\text {env }}\right)$ lists the mass of envelope, Col. (8) ( $\left.M_{\text {disk }}\right)$ lists the mass of disk, Col. (9) $\left(A_{V}\right)$ lists the envelope extinction along the line-of-sight and Col. (10) lists the age.

\begin{tabular}{cccccccccc}
\hline \hline Obj. & $\begin{array}{c}M_{*} \\
\left(M_{\odot}\right)\end{array}$ & $\begin{array}{c}T_{\text {eff }} \\
(\mathrm{K})\end{array}$ & $\begin{array}{c}\text { Luminosity } \\
\left(L_{\odot}\right)\end{array}$ & $\begin{array}{c}\text { Incl. angle } \\
(\mathrm{deg})\end{array}$ & $\begin{array}{c}\dot{M} \\
\left(M_{\odot} / \mathrm{yr}\right)\end{array}$ & $\begin{array}{c}M_{\text {env }} \\
\left(M_{\odot}\right)\end{array}$ & $\begin{array}{c}M_{\text {disk }} \\
\left(M_{\odot}\right)\end{array}$ & $\begin{array}{c}A_{V} \\
(\mathrm{mag})\end{array}$ & $\begin{array}{c}\text { Age } \\
(\mathrm{yr})\end{array}$ \\
\hline G4 Model 1 & 6.7 & 4241 & $5.2 \times 10^{2}$ & 18 & $3.9 \times 10^{-5}$ & 2.4 & $1.6 \times 10^{-2}$ & 19.3 & $5 \times 10^{3}$ \\
G4 Model 2 & 9.2 & 24552 & $4.6 \times 10^{3}$ & 81 & 0 & - & $2.1 \times 10^{-2}$ & 24 & $1 \times 10^{6}$ \\
G5 Model 1 & 10.1 & 4351 & $2.2 \times 10^{3}$ & 18 & $1.1 \times 10^{-3}$ & 47.5 & $4.0 \times 10^{-2}$ & 1960 & $4 \times 10^{3}$ \\
G5 Model 2 & 7.5 & 21770 & $2.3 \times 10^{3}$ & 87 & $1.1 \times 10^{-6}$ & 1.3 & $8.4 \times 10^{-3}$ & 414 & $4 \times 10^{5}$ \\
G5 Model 3 & 7.3 & 21262 & $2.0 \times 10^{3}$ & 87 & $8.6 \times 10^{-9}$ & 0.07 & $2.9 \times 10^{-5}$ & 12 & $1 \times 10^{6}$ \\
G5 Model 4 & 8.1 & 22602 & $2.9 \times 10^{3}$ & 87 & 0 & - & $1.6 \times 10^{-3}$ & $5.5 \times 10^{-4}$ & $4 \times 10^{6}$ \\
\hline
\end{tabular}

The cavity angle in Model 1 for both G4 and G5 is $2^{\circ}-6^{\circ}$ while the cavity angle in Models 2 and 3 of G5 is $40^{\circ}-50^{\circ}$.

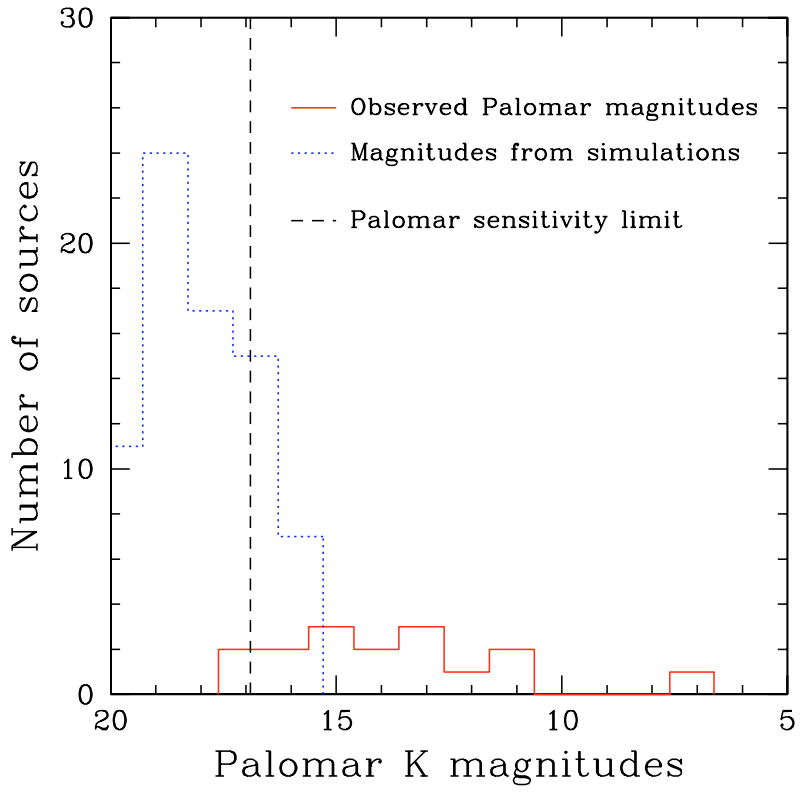

Fig. 10. Plot of magnitude distribution in the $K_{\mathrm{s}}$ band from the simulations and observations (Palomar). The solid line represents the magnitudes of YSOs within the "region of interest" (Table 2). The dotted line represents the apparent magnitudes from one run of model (general case of constant star formation rate with ages between 0.01 and $1 \mathrm{Myr}$ ) simulations. The dashed line denotes the Palomar sensitivity limit.

class II objects. For every cluster member of a certain mass, an age is randomly assigned. If this is less than $0.5 \mathrm{Myr}$, its spectrum is taken to be of the class I type. For ages greater than $0.5 \mathrm{Myr}$, the cluster member is a class II type object.

The median number of cluster members is $\sim 300$ while the median mass of the cluster is $\sim 230 M_{\odot}$. We have also estimated the magnitude distribution from the cluster for the case of a uniform star formation rate (case 3). This is shown for $K_{\mathrm{s}}$ band apparent fluxes in Fig. 10 for the general case incorporating class I and class II objects with ages between 0.01 and $1 \mathrm{Myr}$.

\subsubsection{Comparison with observations}

A comparison of the model results with the observations has been carried out in terms of magnitude distribution (Fig. 10) and number of sources detected (Fig. 11). Figure 10 shows the simulated (case 3) as well as observed magnitude distributions from the Palomar NIR data. The observed sources used in the figure are the young stellar objects detected in the $K_{\mathrm{s}}$ band and listed

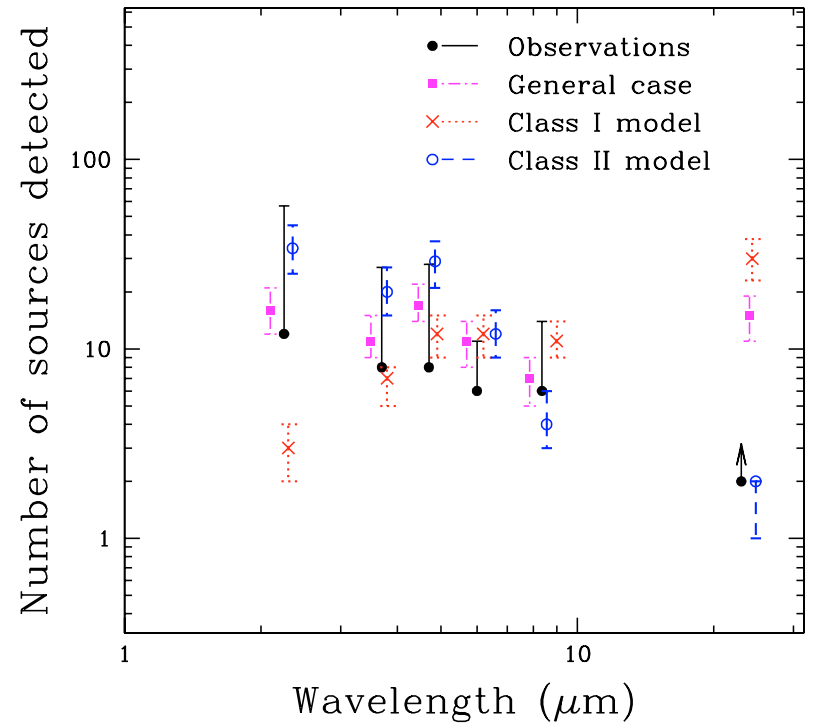

Fig. 11. Plot of the detected number of sources as a function of wavelength for cluster model simulations with observations. The filled circles and solid lines represent the observations and error bars, respectively. The observation at $24 \mu \mathrm{m}$ represents a lower-limit. The solid squares represent median values of the general case of simulations incorporating class I as well as class II objects for the general case of constant star formation rate with ages between 0.01 and $1 \mathrm{Myr}$ and the dot-dashed line represent the quartile values on either side of the median. The cross symbols and dotted line represent the class I model simulations for an age of $0.5 \mathrm{Myr}$. The open circles and dashed line denote class II model simulations for $1 \mathrm{Myr}$. The wavelengths for different cases have been slightly shifted for better viewing. Further details can be found in the text.

in Table 2. Also marked on the figure is the Palomar $K_{\mathrm{s}}$ band sensitivity limit. A comparison of the observed and simulated magnitudes shows that the sources detected in observations are brighter than those simulated. This could be because the density distribution of the cloud is inhomogeneous while the cluster simulations assume a constant density distribution of gas. Another possibility is that the distribution of young stellar objects is more extended than the size of the cloud. From the simulations, it is clear that with the sensitivity limit of Palomar, only a small fraction of sources in the cluster are detected.

From the model, the median number of detected sources in each band has been derived based on the completeness limit of the instrument used for comparing the results. Figure 11 shows the number of detected cluster members predicted by the model in each band. The errorbars indicate the quartile values 
encompassing $50 \%$ of the number of detected cluster members. The cross and dotted line represent case 1 (class I); open circle and dashed line represent case 2 (class II); and, filled square and dot-dashed line represent case 3 (general case incorporating class I class II objects). The observed number of sources (from Palomar image and IRAC1, IRAC2, IRAC3 and IRAC4 bands of GLIMPSE images as well as from MIPS $24 \mu \mathrm{m}$ of MIPSGAL image) are shown by the solid circles and solid lines. The wavelengths for different cases have been slightly shifted for better viewing.

The observed number of sources in each band is shown by a solid line depicting a range of possible number of cluster members, between the lower and upper limits. The lower limit is obtained from the number of young stellar objects detected in that band within the "region of interest" by using the colour-colour diagrams (ref Table 2). The upper edge of each solid line represents the total number of sources detected within that band. For the case of the MIPS $24 \mu \mathrm{m}$ band, there are only two sources detected within the region of interest. IRAS 18511 B shows an extension at $24 \mu \mathrm{m}$ and therefore we have plotted the number of sources detected as 2. Obviously, this represents a lower limit as the sources are unresolved due to lower angular resolution.

From Fig. 11, we find that among all the three cases, the general case incorporating class I $(\sim 50 \%)$ and class II $(\sim 50 \%)$ sources agrees well with the observations. The models with class I and class II sources alone are inconsistent with the observational data. We, therefore infer that the sources in IRAS 18511 are very young and deeply embedded. In $K_{\mathrm{s}}$ as well as in IRAC1 bands, the general case (case 3 ) as well as class II (case 2) model results are consistent with observations. This is expected as more class II sources should be detected in the $K_{\mathrm{s}}$ band. However, in the IRAC2 and IRAC3 bands, all the model results agree with observations within the quartile values. For the IRAC4 band, the class I as well as the general case agree with observations better than with the class II case. Finally, at $24 \mu \mathrm{m}$, the number of detected sources from the class I model and the general case are higher than that detected from the class II model. It must be noted that the observations at $24 \mu \mathrm{m}$ represent a lower limit due to the low angular resolution of the MIPS instrument $\left(\sim 6^{\prime \prime}\right)$. Among all the cases, the general case (case 3) assuming a mix of $\sim 50 \%$ class I and $\sim 50 \%$ class II sources can be said to be a reasonable fit to observations. The model suffers from a few limitations which are listed below.

- The cluster is embedded in a cloud of gas which is homogeneous and clumpiness/density distributions has not been taken into consideration.

- The number of detected cluster members from the simulations (which are point-like) is compared with the observed number of sources. A better comparison would be possible if the results of the simulations were convolved with the beam size of the instrument.

\section{Discussion}

\subsection{Clustering}

We have attempted to study the clustering of the sources around IRAS 18511 using the NIR Palomar images. The method used is described in detail by Testi et al. (1998) to study the clustering around Herbig Ae/Be stars. A density profile is computed centred on the bright IRAS 18511 A source to look for evidence of clustering. Density profiles are computed using the Palomar $K_{\mathrm{s}}$, IRAC2 $(4.5 \mu \mathrm{m})$ and IRAC4 $(8 \mu \mathrm{m})$ band sources as shown

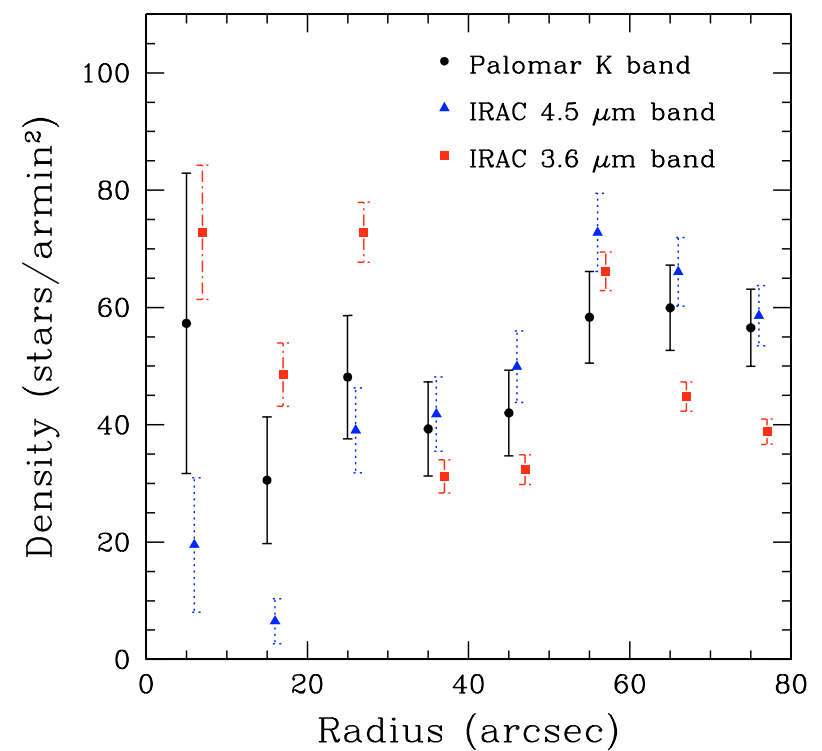

Fig. 12. The clustering indicator (radial profile) centred on the bright NIR source in IRAS 18511, constructed using the Palomar $K_{\mathrm{s}}$ (solid circles), IRAC $4.5 \mu \mathrm{m}$ (solid triangles) and IRAC $8.0 \mu \mathrm{m}$ (solid squares) band fluxes of the sources in the region around IRAS 18511. The star densities derived for the IRAC2 and IRAC4 bands have been scaled by factors of 1.7 and 6.4, respectively for suitable comparison with the radial profile in the $K_{\mathrm{s}}$ band. The radii for different cases have been slightly shifted for better viewing.

in Fig. 12. In this plot, the star densities derived for the IRAC2 and IRAC4 bands have been scaled by factors of 1.7 and 6.4 , respectively for comparison with the radial profile derived for the $K_{\mathrm{s}}$ band. Further, the radii of the annuli of the IRAC bands are slightly shifted for better viewing of the plot. From the profile, we do not see any "clear" evidence for clustering centred on A. This is consistent with the results of the previous sections, i.e. the extinction due to the associated molecular cloud is rather high and there may be other embedded sources. This is consistent with the results of our simulations, i.e. only a small fraction of sources are detected.

\subsection{Extinction at $8.0 \mu \mathrm{m}$ vs. $850 \mu \mathrm{m}$}

A comparison of the morphology of the sub-millimetre dust emission and the filamentary structures seen in the $8.0 \mu \mathrm{m}$ emission map indicates that these filamentary structures comprise of cold dust. Figure 3 shows the $8.0 \mu \mathrm{m}$ grayscale image with $850 \mu \mathrm{m}$ contours overlaid. The morphology of the $850 \mu \mathrm{m}$ emission traces the filamentary structures close to IRAS 18511. It is evident that these filaments represent the cold dust comprising the infrared dark cloud with IRAS 18511 located at the peak of one clump.

We have compared the extinction values obtained from the JCMT-SCUBA $850 \mu \mathrm{m}$ image (using Eq. (2)) with that from IRAC $8.0 \mu$ m image (using Eq. (3)). Resampling the images and a pixel-to-pixel comparison of the extinction values in the region of overlap of the filament (to the south-west of IRAS $18511 \mathrm{~A}$ ) shows that the visual extinction values obtained using the JCMTSCUBA sub-millimetre image is $\sim 8$ times the the $A_{\mathrm{V}}$ values obtained from the IRAC $8.0 \mu \mathrm{m}$ image. However the $A_{\mathrm{V}}$ values quoted for the IRAC $8.0 \mu \mathrm{m}$ image are lower limits for the following reason. Equation (3) assumes the emitting dust to be behind the absorbing filaments and this is clearly not correct since $I_{0}$ has contribution from foreground emission. Consequently, the 
values of $A_{\mathrm{V}}$ derived are an underestimate. And also, the mass estimates derived for the filaments and the clump in Sect. 3.2.1 are lower-limits. Brand et al. (2001) have imaged a number of molecular lines in the IRAS 18511 region $\left({ }^{13} \mathrm{CO}, \mathrm{CS}, \mathrm{HCO}^{+}\right)$. Of particular interest is the $\mathrm{HCO}^{+}$emission integrated over the red part of the emission. This emission extends along the direction of the filaments to the south-west, as seen in the JCMT-SCUBA $850 \mu \mathrm{m}$ image. Further, the emission is at a velocity $\left(V_{\mathrm{lsr}}\right)$ of $\sim 59 \mathrm{~km} \mathrm{~s}^{-1}$. This is the $V_{\text {lsr }}$ of IRAS 18511 and hence, the filamentary structures are associated with the molecular cloud of IRAS 18511.

Using the fact that the $A_{V}$ values derived from the $850 \mu \mathrm{m}$ sub-millimetre map is $\sim 8$ times larger than that obtained from the IRAC $8.0 \mu \mathrm{m}$ map, we can estimate the fraction of foreground emission contributing to the total emission detected (in the neighbourhood of the filaments). We estimate that this fraction of foreground emission is $\sim 80 \%$ of the detected emission. In other words, most of the emission is foreground emission. This is not surprising considering that IRAS 18511 is a distant object ( 3.9 kpc).

\subsection{Evolutionary stage of cluster members}

It is interesting to compare the multiwavelength observations of $\mathrm{A}$ and $\mathrm{B}$, although each comprises of more than one source. $\mathrm{A}$ is the brightest source in the near, mid and far infrared as well as in the sub-millimetre wavebands. However, there is no detected radio emission associated with $\mathrm{A}$. On the other hand, G5 (associated with $\mathrm{B}$ ) has free-free radio emission associated with it. The peak of molecular line $\left(\mathrm{C}^{18} \mathrm{O}\right.$ and $\left.{ }^{13} \mathrm{CO}\right)$ emission, as observed by Watt \& Mundy (1999) is located near B and one can see an extension corresponding to $\mathrm{A}$. The absence of radio emission near A and other observations suggest the following possibilities: (1) A is in an earlier evolutionary stage than B and the ultracompact $\mathrm{H}$ II region has not yet formed. This would explain the absence of radio emission as well as their brightness at infrared wavebands (2) A being more massive than B, its ultracompact $\mathrm{H}$ II region is being choked by the infalling matter through accretion (Keto 2003). Kurtz et al. (2004) have carried out a VLA survey in the $44 \mathrm{GHz}$ (class I) methanol maser line in several star forming regions including IRAS 18511. They find a maser in the vicinity of IRAS 18511 A (synthesized beam size $\sim 2$ '. $2 \times 1$ '. 5 ). The position of the maser (positional uncertainy $\sim 0.5$ ) is shown in Fig. 2 . They also find in their survey that class I methanol masers are typically offset by $\sim 0.2 \mathrm{pc}$ (median value) from other massive star formation signposts like H II regions or water masers. Cyganowski et al. (2007) have studied the massive protocluster associated with $\mathrm{S} 255 \mathrm{~N}$ and they find that the class I methanol are aligned in the direction of the outflow. In the case of IRAS 18511, the methanol maser is at distance of $0.1 \mathrm{pc}$ from IRAS $18511 \mathrm{~A}$ and $0.4 \mathrm{pc}$ from G5 (VLA source). Also, no water masers or H II region have been detected near IRAS 18511 A. Further, it is believed that shocked gas from outflows/accretion might be responsible for class I methanol masers (Plambeck \& Menten 1990; Johnston et al. 1992). Zhang et al. (2005) have imaged IRAS $18511\left(\sim 30^{\prime \prime}\right)$ in the CO $(J=2 \rightarrow 1)$ line and the emission shows a peak close to IRAS $18511 \mathrm{~A}$. This is unlike the case of $\mathrm{C}^{18} \mathrm{O}$ where the peak of emission is located at IRAS 18511 B (Watt \& Mundy 1999). There is also evidence for an outflow from IRAS 18511 A since wings are seen in the line profiles observed by Brand et al. (2001). All these facts suggest that IRAS $18511 \mathrm{~A}$ is in an earlier evolutionary state than IRAS $18511 \mathrm{~B}$.
Considering the above observations, analyses and simulations of the cluster associated with IRAS 18511, the following likely scenario emerges. This is a young cluster with sources in an early evolutionary phase (class I and class II). The large extinction indicates that probably there are more low-mass cluster members hidden in the molecular cloud which will start appearing as the cluster evolves and the molecular cloud disperses. IRAS $18511 \mathrm{~A}$ is likely to be an intermediate mass/massive young object, probably a class I object. Its luminosity indicates that it is probably the most massive object in the cluster. On the other hand, the presence of radio emission near G5 indicates that G5 could be an older member of the cluster. The absence of other young objects in the vicinity of IRAS 18511 A can possibly be explained by large extinction as indicated by the JCMT sub-millimetre maps (peak of emission is at A). Further study of IRAS 18511 (high angular resolution mid infrared maps and molecular line maps) can shed light on the likely scenario.

\subsection{Precursor to Herbig star type cluster?}

The clusters associated with Herbig stars and their properties have been studied by Testi et al. (1997), Testi et al. (1998), Testi et al. (1999). In particular, the mid infrared emission $(10 \mu \mathrm{m})$ from a few such clusters has been investigated by Habart et al. (2003). The luminosity as well as the clustering suggests that IRAS 18511 could be a precursor to a Herbig cluster. Testi et al. (1999) report the tendency of early Herbig Be stars to be surrounded by dense clusters of lower mass companions. Some of the clusters associated with earlier Herbig stars (of ZAMS spectral type B0) are found to have $\sim 75$ members, where the lower mass limit is $\sim 0.2 M_{\odot}$ for 2 mag of extinction in $\mathrm{K}$. It is interesting to compare these numbers with the model results which suggest cluster membership to be $\sim 200$ for masses greater than $0.2 M_{\odot}$. While the model results predict the number of cluster members to be $\sim 2-3$ times the number observed by Testi et al. (1999), it is useful to note that the clusters of Testi et al. (1999) are more evolved as compared to the model which is an embedded cluster. And evolved clusters with intermediate mass Herbig Ae/Be stars retain less than $50 \%$ of its members (Weidner et al. 2007) Further, the size of the embedded cluster (size of cloud from sub-millimetre map) associated with IRAS 18511 is $\sim 0.6 \mathrm{pc}$. This compares well with the cluster sizes of $0.2-0.7 \mathrm{pc}$ obtained for clusters associated with Herbig Ae/Be stars of spectral type B0 (Testi et al. 1999). The mid infrared (excess) emission as well as the large extinction (it is not visible optically) indicates that the main source (A) is in an early evolutionary stage. Habart et al. (2003) find objects with mid infrared emission from circumstellar disks and envelopes in five out of twelve fields of known Herbig AeBe stars they studied. The IRAS 18511 field also shows many objects in mid infrared. In short, the following considerations: (a) luminosity (b) evolutionary stage of the objects (c) size of associated cluster and (d) number of cluster members, suggest that the source associated with IRAS 18511 is a protocluster associated with a candidate precursor to a Herbigstar cluster. This is in accordance with the suggestions of Testi et al. (1998) and Molinari et al. (2000). We therefore, believe that IRAS 18511 represents an early stage of clusters associated with an intermediate mass object.

\section{Summary}

With the aim of studying the early evolution of clusters, we have selected IRAS 18511 to carry out a detailed investigation. Using 
emission at sub-millimetre (JCMT-SCUBA), infrared (SpitzerMIPS, Spitzer-IRAC and Palomar) and radio (VLA) wavelengths, we have studied the main source as well as the cluster associated with IRAS 18511. The results are consistent with simulations of a young embedded cluster incorporating class I and class II sources. Based on the luminosity, and properties of the associated cluster (number of members, size of cluster and evolutionary stages of objects), we conclude that IRAS 18511 is a protocluster with the most massive object being a candidate precursor to a Herbig Ae/Be star.

Acknowledgements. We would like to thank R. Cesaroni for his help and suggestions.

\section{References}

Allen, L. E., Calvet, N., D’Alessio, P., et al. 2004, ApJS, 154, 363

Baraffe, I., Chabrier, G., Allard, F., \& Hauschildt, P. H. 2002, A\&A, 382, 563

Benjamin, R. A., Churchwell, E., Babler, B. L., et al. 2003, PASP, 115, 953

Bianchi, S., Gonçalves, J., Albrecht, M., et al. 2003, A\&A, 399, L43

Brand, J., Cesaroni, R., Palla, F., \& Molinari, S. 2001, A\&A, 370, 230

Carey, S. J., Noriega-Crespo, A., Price, S. D., et al. 2005, in BAAS, 1252

Cohen, M., \& Schwartz, R. D. 1983, ApJ, 265, 877

Cyganowski, C. J., Brogan, C. L., \& Hunter, T. R. 2007, ArXiv e-prints, 704

Fazio, G. G., Hora, J. L., Allen, L. E., et al. 2004, ApJS, 154, 10

Furlan, E., Hartmann, L., Calvet, N., et al. 2006, ApJS, 165, 568

Habart, E., Testi, L., Natta, A., \& Vanzi, L. 2003, A\&A, 400, 575

Hildebrand, R. H. 1983, QJRAS, 24, 267

Hunt, L. K., Mannucci, F., Testi, L., et al. 1998, AJ, 115, 2594

Indebetouw, R., Mathis, J. S., Babler, B. L., et al. 2005, ApJ, 619, 931
Ishii, M., Nagata, T., Chrysostomou, A., \& Hough, J. H. 2002, AJ, 124, 2790

Johnston, K. J., Gaume, R., Stolovy, S., et al. 1992, ApJ, 385, 232

Keto, E. 2003, ApJ, 599, 1196

Kurtz, S., Hofner, P., \& Álvarez, C. V. 2004, ApJS, 155, 149

Lada, C. J., \& Adams, F. C. 1992, ApJ, 393, 278

Lada, C. J., \& Lada, E. A. 2003, ARA\&A, 41, 57

Makovoz, D., Marleau, F. R., \& Frayer, D. T. 2004, in BAAS, 1604

Meyer, M. R., Calvet, N., \& Hillenbrand, L. A. 1997, AJ, 114, 288

Molinari, S., Brand, J., Cesaroni, R., \& Palla, F. 1996, A\&A, 308, 573

Molinari, S., Brand, J., Cesaroni, R., Palla, F., \& Palumbo, G. G. C. 1998, A\&A, 336, 339

Molinari, S., Brand, J., Cesaroni, R., \& Palla, F. 2000, A\&A, 355, 617

Palla, F., \& Stahler, S. W. 1999, ApJ, 525, 772

Panagia, N. 1973, AJ, 78, 929

Persson, S. E., Murphy, D. C., Krzeminski, W., Roth, M., \& Rieke, M. J. 1998, AJ, 116, 2475

Plambeck, R. L., \& Menten, K. M. 1990, ApJ, 364, 555

Rieke, G. H., \& Lebofsky, M. J. 1985, ApJ, 288, 618

Rieke, G. H., Young, E. T., Engelbracht, C. W., et al. 2004, ApJS, 154, 25

Robitaille, T. P., Whitney, B. A., Indebetouw, R., \& Wood, K. 2007, ApJS, 169, 328

Schraml, J., \& Mezger, P. G. 1969, ApJ, 156, 269

Testi, L., Palla, F., \& Natta, A. 1998, A\&AS, 133, 81

Testi, L., Palla, F., \& Natta, A. 1999, A\&A, 342, 515

Testi, L., Palla, F., Prusti, T., Natta, A., \& Maltagliati, S. 1997, A\&A, 320, 159

Wang, S., \& Looney, L. W. 2007, ApJ, 659, 1360

Watt, S., \& Mundy, L. G. 1999, ApJS, 125, 143

Weidner, C., Kroupa, P., Nürnberger, D. E. A., \& Sterzik, M. F. 2007, MNRAS, 376,1879

Werner, M. W., Roellig, T. L., Low, F. J., et al. 2004, ApJS, 154, 1

Whittet, D. C. B. 1992, J. British Astron. Assoc., 102, 230

Zhang, Q., Hunter, T. R., Brand, J., et al. 2005, ApJ, 625, 864 
S. Vig et al.: IRAS 18511+0146: a proto Herbig Ae/Be cluster?, Online Material $p$ I

\section{Online Material}




\section{Appendix A: Photometry of Spitzer images}

IRAS 18511 consists of a very bright source and a small group of stars near it. We, therefore, first explored our photometry techniques on a field consisting of isolated sources (which are not saturated) with good signal-to-noise. The following methods were used.

1. APEX - The Spitzer Astronomical Point Source EXtraction (APEX) is a software provided by the Spitzer Science Centre for point source extraction and photometry. We have used the Linux version MOPEX 030106 for our study. In this software, the sources are first detected using non-linear matched filtering and image segmentation. Subsequent point source extraction is performed by fitting the Point Response Function (PRF) to the detected sources (Makovoz et al. 2004). More details can be found in the APEX Manual. For PRF fitting, the PRF has been taken from the Spitzer Science Center (SSC) webpage. In addition, aperture photometry can also be carried out. We have compared the fluxes from apertures of radii $6^{\prime \prime}, 9^{\prime \prime}$ and $12^{\prime \prime}$.

2. DAOPHOT - We have used the IDL version of the IRAFDAOPHOT package in order to carry out source extraction and aperture photometry with an aperture radius of $6^{\prime \prime}$ and background annulus of $12^{\prime \prime}$. Appropriate aperture corrections have been applied.

3. SExtractor - Source Extractor is a program which can be used to extract sources from moderately crowded fields. The background in this package is, however, determined from the full image and the objects above a given threshold are detected and de-blended. For photometry, we have used an aperture radius of $6^{\prime \prime}$.

4. AIPS/GILDAS - We have also used the AIPS/GILDAS software to compute the integrated flux down to $10 \%$ of the peak value after subtracting the background. Appropriate aperture corrections have been applied depending on the size of the integration area.

All the above methods did a reasonably good task of source extraction and photometry. The values of fluxes of the isolated bright sources obtained from the methods mentioned above were compared. The values were found to be within $5 \%$ of each other. We then proceeded to extract and carry out photometry of the sources in the region around IRAS 18511 which has bright saturated sources located close to each other as well as some diffuse emission. The saturated pixels were replaced by an average value of the neighbouring non-saturated pixels. For this region, we find the following:

- The source extraction by SEXTRACTOR is better than that by the PRF fitting methods, APEX and DAOPHOT.

- The aperture photometry methods yield consistent results within $5 \%$ of the flux values.

- The PRF fitting method of APEX finds quite a large number of sources near the bright source and the flux is distributed among them.

- The flux values obtained by AIPS/GILDAS which integrates fluxes in circular areas down to $10 \%$ of the peak brightness after subtracting the background gives consistent results with those from aperture photometry.

\section{Appendix B: Description of the cluster simulations}

For a given IMF (Scalo or Salpeter or Kroupa), the cluster members are randomly selected by using a Monte-Carlo method. The lower mass limit is taken to be $0.1 M_{\odot}$. An age (star formation hisory) is assigned to each cluster member according to either of the two prescriptions: (a) coeval formation - every cluster member has the same age, or (b) uniform formation rate - an age is assigned to every cluster member which falls randomly between the ages representing the start and finish of the star formation process. The luminosity and effective temperature of every cluster member having a certain mass and age is determined using the pre-main sequence tracks of Palla \& Stahler (1999). Because of uncertainties in the early pre-main sequence evolution of stars, we have assumed a lower limit on the age. The pre main sequence track corresponding to $0.5 \mathrm{Myr}$ is applied even to objects with ages less than $0.5 \mathrm{Myr}$. For ages of a given mass (as well as masses for a given age) greater than that for the pre-main sequence tracks, the luminosities and effective temperatures have been obtained by assuming them to be lying on the tracks of zero-age main sequence (ZAMS) stars. For such class II objects, it is assumed that although the central source has reached the ZAMS stage, there is a remnant disk around it. The cluster members are randomly added to the cluster till the total luminosity reaches the bolometric luminosity of the cluster. It is important to note that the the luminosity of each cluster member includes luminosities from the central object (photosphere for the class II objects) as well as from the circumstellar material (disk for class II and envelope for class I objects).

The cluster members are now placed at random locations in a homogeneous spherical cloud of gas of assumed mass and size (derived from the sub-millimetre/millimetre maps). In other words, the distribution of the cluster members follows the gas distribution. Thus, there is a column of gas in front of every embedded cluster member which determines its extinction. Therefore, different cluster members suffer different amounts of extinctions based on their positions within the cloud of gas. An additional visual extinction of $7 \mathrm{mag}$ (due to ISM) has been added to the extinctions. Taking the total extinction into consideration, the apparent magnitude of every cluster member is determined in each band and is considered detected if the object is brighter than the sensitivity limit in that band. The sensitivities in various bands as well as the extinction laws used are listed in Table B.1. The near infrared extinction laws have been taken from Rieke \& Lebofsky (1985). For the Spitzer-IRAC bands, we have used the average extinction value obtained by Indebetouw et al. (2005). At $24 \mu \mathrm{m}$, we have used an extrapolated extinction value.

Every cluster member is classified as class I or class II. Sources younger than $0.5 \mathrm{Myr}$ are considered as class I and those older than $0.5 \mathrm{Myr}$ are considered to be of class II type. We have taken the age of 0.5 Myr between the class I and more evolved objects as the pre-main sequence evolution models show uncertainities below the age of 1 Myr (Baraffe et al. 2002). For class I sources, the flux in various bands has been determined based on the spectrum of the well-studied class I type low mass protostar, L1551-IRS5. Cohen \& Schwartz (1983) list the magnitudes of L1551-IRS5 in 11 bands ranging from $1.2 \mu \mathrm{m}$ to $19 \mu \mathrm{m}$. The ratio of the absolute flux (corrected for distance of L1551) to the luminosity of L1551-IRS5 $\left(\sim 35 L_{\odot}\right)$ has been used as a scaling factor to obtain the fluxes of class I type objects in the simulations corresponding to the luminosities of these objects. The ratios of fluxes (in various bands) to the total luminosity are tabulated in Table B.1.

In order to determine the emission from pre-main sequence objects in the class II phase, we have used the median spectrum of class II type objects in the Taurus star forming region (Table 6 of Furlan et al. 2006). This median spectrum has been 
Table B.1. Extinction laws and various other constants used in the simulations. $F_{\lambda} / F_{H}$ represents the ratio of flux in each band with respect to the flux in $H$ band assumed for class II sources in the simulations based on the median spectrum of class II sources by Furlan et al. (2006) and $\lambda / L$ represents the ratio of flux in each band to the luminosity of L1551IRS5 (Cohen \& Schwartz 1983) used to estimate the fluxes of class I sources in the simulations.

\begin{tabular}{ccccc}
\hline \hline$\lambda$ & $A_{\lambda} / A_{V}$ & $F_{\lambda} / F_{H}$ & $\lambda / L$ & Sensitivity limit \\
\hline$\mu \mathrm{m}$ & & & $\left(\mu \mathrm{m} / L_{\odot}\right)$ & \\
\hline 2.2 & 0.112 & 0.979 & 0.685 & 16.9 \\
3.5 & 0.063 & 0.787 & 2.107 & 14.5 \\
4.6 & 0.048 & 0.714 & 9.880 & 14.0 \\
5.8 & 0.048 & 0.645 & 14.65 & 13.0 \\
8 & 0.048 & 0.654 & 27.51 & 11.5 \\
24 & 0.005 & 1.326 & 495.0 & 6.8 \\
\hline
\end{tabular}

constructed using fluxes from bands including the near infrared $\left(J H K_{\mathrm{s}}\right)$ and Spitzer-IRAC $(3.6,4.5,5.8$ and $8.0 \mu \mathrm{m})$ bands. This median spectrum implies flux ratios (flux in various bands with respect to the flux in the $H$-band) which are given in Table B. 1 . The normalization has been carried out with respect to the flux values in $H(1.65 \mu \mathrm{m})$ band since the $H$-band flux is photospheric for most class II objects (Furlan et al. 2006). As the class I and class II spectra are of low-mass objects, we have used these in the model simulations for objects of all masses. Although the pre-main sequence evolution of massive stars is not clearly understood, we use these class I and class II spectra since fits from RWIW models (Sect. 3.6.1) indicate that the SEDs of cluster members of IRAS 18511 are well-fit by models comprising massive central objects. The class I and class II type spectral energy distributions, used in the model, are shown in Fig 8. These spectra have been normalised with respect to the $H$ $(1.65 \mu \mathrm{m})$ band. The Spitzer colours of class I and class II SEDs considered for modelling are shown in Fig. 4. 